\title{
Probing supersymmetry with parity-violating electron scattering
}

\author{
A. Kurylov \\ California Institute of Technology, Pasadena, California 91125, USA \\ M. J. Ramsey-Musolf \\ California Institute of Technology, Pasadena, California 91125, USA, \\ Department of Physics, University of Connecticut, Storrs, Connecticut 06269, USA, \\ and Institute for Nuclear Theory, University of Washington, Seattle, Washington 98195, USA \\ S. Su \\ California Institute of Technology, Pasadena, California 91125, USA \\ (Received 13 March 2003; published 15 August 2003)
}

\begin{abstract}
We compute the one-loop supersymmetric (SUSY) contributions to the weak charges of the electron $\left(Q_{W}^{e}\right)$, proton $\left(Q_{W}^{p}\right)$, and cesium nucleus $\left(Q_{W}^{\mathrm{Cs}}\right)$ in the minimal supersymmetric standard model (MSSM). Such contributions can generate several percent corrections to the corresponding standard model values. The magnitudes of the SUSY loop corrections to $Q_{W}^{e}$ and $Q_{W}^{p}$ are correlated over nearly all of the MSSM parameter space and result in an increase in the magnitudes of these weak charges. In contrast, the effects on $Q_{W}^{\text {Cs }}$ are considerably smaller and are equally likely to increase or decrease its magnitude. Allowing for $R$-parity violation can lead to opposite sign relative shifts in $Q_{W}^{e}$ and $Q_{W}^{p}$, normalized to the corresponding standard model values. A comparison of $Q_{W}^{p}$ and $Q_{W}^{e}$ measurements could help distinguish between different SUSY scenarios.
\end{abstract}

DOI: 10.1103/PhysRevD.68.035008

PACS number(s): 12.60.Jv, 11.30.Er, 12.15.Lk, 13.60.Hb

\section{INTRODUCTION}

The search for physics beyond the standard model (SM) of electroweak and strong interactions is a primary objective for particle and nuclear physics. Historically, parity-violating (PV) interactions have played an important role in elucidating the structure of the electroweak interaction. In the 1970s, PV deep inelastic scattering (DIS) measurements performed at the Stanford Linear Accelerator Center (SLAC) confirmed the SM prediction for the structure of weak neutral current interactions [1]. These results were consistent with a value for the weak mixing angle given by $\sin ^{2} \theta_{W} \approx 1 / 4$, implying a tiny $V$ (electron) $\times A$ (quark) neutral current interaction. Subsequent PV measurements-performed at both very low scales using atoms as well as at the $Z$ pole in $e^{+} e^{-}$ annihilation-have been remarkably consistent with the results of the SLAC DIS measurement [1].

More recently, the results of cesium atomic parityviolation (APV) [2] and deep inelastic $\nu$ - $(\bar{\nu}-)$ nucleus scattering [3] have been interpreted as determinations of the scale dependence of $\sin ^{2} \theta_{W}$. The SM predicts how this quantity should depend on the momentum transfer squared $\left(q^{2}\right)$ of a given process. ${ }^{1}$ The cesium APV result appears to be consistent with the SM prediction for $q^{2} \approx 0$, whereas the neutrino DIS measurement implies a $+3 \sigma$ deviation at $\left|q^{2}\right|$ $\sim 20(\mathrm{GeV} / c)^{2}$. If conventional hadron structure effects are

\footnotetext{
${ }^{1}$ The weak mixing angle and its $q^{2}$ evolution are renormalization scheme-dependent. Here, we use the modified minimal subtraction $(\overline{\mathrm{MS}})$ scheme for the SM and the dimensional reduction $(\overline{\mathrm{DR}})$ scheme for supersymmetric extensions of the SM.
}

ultimately unable to account for the NuTeV "anomaly," the results of this precision measurement would point to new physics.

In light of this situation, two new measurements involving polarized electron scattering have taken on added interest: PV Möller (ee) scattering at SLAC [4] and elastic, PV ep scattering at the Jefferson Lab (JLab) [5]. In the absence of new physics, both measurements could be used to determine $\sin ^{2} \theta_{W}$ at the same scale $\left[\left|q^{2}\right| \approx 0.03(\mathrm{GeV} / c)^{2}\right]$-falling between the scales relevant to the APV and neutrino DIS measurements-with comparable precision in each case $^{2}$ $\left(\Delta \sin ^{2} \theta_{W} \approx 7 \times 10^{-4}\right.$ ). Any significant deviation from the SM prediction for $\sin ^{2} \theta_{W}$ at this scale would provide striking evidence for new physics, particularly if both measurements report a deviation. On the other hand, agreement would imply that the most likely explanation for the neutrino DIS result involves hadron structure effects within the SM.

In this paper, we analyze the prospective implications of the parity-violating electron scattering (PVES) measurements for supersymmetry (SUSY). Although no supersymmetric particle has yet been discovered, there exists strong theoretical motivation for believing that SUSY is a component of the "new" standard model. For example, the existence of low-energy SUSY is a prediction of many string theories; it offers a solution to the hierarchy problem; and it results in coupling unification close to the Planck scale. In addition, if $R$ parity is conserved (see below), SUSY provides an excellent candidate for cold dark matter, the lightest neutralino (see Ref. [7] for a review). The existence of such

\footnotetext{
${ }^{2}$ In practice, the PV ep experiment will actually provide a value for $\sin ^{2} \theta_{W}\left(q^{2}=0\right)$, as discussed in Ref. [6].
} 
dark matter is required in most cosmological models. In light of such arguments, it is clearly of interest to determine what insight about SUSY, if any, the new PVES measurements might provide.

In the simplest version of SUSY - the minimal supersymmetric standard model (MSSM) with conserved $R$ parity [8] - low-energy precision observables experience SUSY only via tiny loop effects involving virtual supersymmetric particles. The requirement of baryon minus lepton number $(B-L)$ conservation leads to conservation of the $R$-parity quantum number, $P_{R}=(-1)^{2 S+3(B-L)}$, where $S$ denotes spin. Every SM particle has $P_{R}=+1$ while the corresponding superpartner, whose spin differs by $1 / 2$ unit, has $P_{R}=$ -1 . Conservation of $P_{R}$ implies that every vertex has an even number of superpartners. Consequently, for processes like $e e \rightarrow e e$ and $e p \rightarrow e p$, all superpartners must live in loops, which generate corrections-relative to the SM amplitude-of order $(\alpha / \pi)(M / \widetilde{M})^{2} \sim 10^{-3}$ (where $M$ denotes a SM particle mass and $\tilde{M}$ is a superpartner mass). Generally speaking, then, low-energy experiments must probe an observable with a precision of few tenths of a percent or better in order to discern SUSY loop effects. Lowenergy charged current experiments have already reached such levels of precision, and the corresponding implications of these experiments for the MSSM have been discussed elsewhere [9].

In the case of PV ee and elastic ep scattering, the precision needed to probe SUSY loop effects is roughly an order of magnitude less stringent, owing to a fortuitous suppression of the SM PV asymmetries, $A_{L R}$. At leading order in $q^{2}$, the $A(e) \times V(f)$ contributions to $A_{L R}$ are governed by $Q_{W}^{f}$, the "weak charge" of the target fermion, $f$. The weak charge of a particle $f$ is defined as the strength of the effective $A(e) \times V(f)$ interaction:

$$
\mathcal{L}_{E F F}^{e f}=-\frac{G_{\mu}}{2 \sqrt{2}} Q_{W}^{f} \bar{e} \gamma_{\mu} \gamma_{5} e \bar{f} \gamma^{\mu} f .
$$

At tree level in the SM the weak charges of both the electron and the proton are suppressed: $Q_{W}^{p}=-Q_{W}^{e}=1-4 \sin ^{2} \theta_{W}$ $\approx 0.1$. One-loop SM electroweak radiative corrections further reduce this tiny number, leading to the predictions $Q_{W}^{e}$ $=-0.0449[6,10]$ and $Q_{W}^{p}=0.0716$ [6]. The factor of $\gtrsim 10$ suppression of these couplings in the SM renders them more transparent to the possible effects of new physics. Consequently, experimental precision of order a few percent, rather than a few tenths of a percent, is needed to probe SUSY loop corrections. (Theoretical uncertainties associated with QCD corrections to $Q_{W}^{e, p}$ are considerably smaller $[6,10]$.)

In analyzing these SUSY loop contributions to $Q_{W}^{e, p}$, we carry out a model-independent treatment, avoiding the choice of a specific mechanism for SUSY-breaking mediation. While most analyses of precision electroweak observables have been performed using one or more widely used models for SUSY-breaking mediation, the generic features of the superpartner spectrum implied by such models may not be consistent with precision data [9]. Consequently, we wish to determine the possible impact of SUSY on the two PVES measurements for all phenomenologically acceptable choices of the MSSM parameters, even if such choices lie outside the purview of standard SUSY-breaking models. In doing so, we follow the spirit of Ref. [11], where a similar analysis of SUSY loop effects in $\nu(\bar{\nu})$-nucleus scattering was performed.

In the case of PV electron scattering, we find that the magnitude of SUSY loop effects could be as large as the proposed experimental uncertainties for the $Q_{W}^{e}$ and $Q_{W}^{p}$ measurements $(8 \%$ and $4 \%$, respectively $[4,5])$. Moreover, the relative sign of the effect (compared to the SM prediction) in both cases is correlated - and positive-over nearly all available SUSY parameter space. To our knowledge, such correlation is specific to the MSSM (with $R$-parity conservation), making it a potential low-energy signature of this new physics scenario. We also find that the SUSY loop effects on $Q_{W}^{\mathrm{Cs}}$, the weak charge of the cesium atom measured in APV, are much less pronounced. Thus, the present agreement between the experimental value for $Q_{W}^{\mathrm{Cs}}$ and the SM prediction does not preclude the presence of relatively large effects in the PV electron scattering asymmetries.

We also investigate a scenario where $P_{R}$ is not conserved. We find that, in contrast to the $P_{R}$-conserving SUSY, the relative sign of the effect (compared to the SM prediction) is always negative for $Q_{W}^{e}$ and can have either sign for $Q_{W}^{p}$, with the positive sign being somewhat more likely than the negative sign. The potential magnitude of the effects are considerably larger than those generated by SUSY loops. In principle, then, a comparison of $Q_{W}^{e}$ and $Q_{W}^{p}$ can potentially establish whether or not $R$ parity is violated within a SUSY extension of the SM. Having an answer to this question would have consequences reaching beyond the realm of accelerator physics. For instance, if $P_{R}$ is violated in PVES, then the lepton number is not conserved, thereby implying that neutrinos have Majorana masses and making neutrinoless double beta decay possible (see, e.g. Ref. [12]). $R$-parity violation also renders the lightest supersymmetric particle unstable, thus eliminating SUSY dark matter, which has significant implications for cosmology [7].

Our discussion of these points is organized as follows. After briefly reviewing the minimal supersymmetric standard model in Sec. II, we discuss the structure of the one-loop radiative corrections to $Q_{W}^{e, p}$ in Sec. III and the tree-level $P_{R}$-violating contributions in Sec. IV. The analysis of the prospective implications of the parity-violating electron scattering measurements for supersymmetry is presented in Sec. V. We conclude in Sec. VI. Appendix A lists the counterterms for the effective PVES Lagrangian of Eq. (1) necessary for renormalization of the one-loop radiative corrections to the weak charges. In Appendix B we explicitly prove that gluino loops do not contribute to $Q_{W}^{p}$. In Appendix C we give complete expressions for all process-dependent one-loop SUSY corrections to the $e p$ and ee scattering; expressions for process-independent contributions are given in the appendixes of Ref. [11].

\section{MSSM PARAMETERS}

The content of the MSSM has been described in detail elsewhere [8], so we review only a few features here. The 
particle spectrum consists of the SM particles and the corresponding superpartners: spin-0 sfermions $(\widetilde{f}$, which include sneutrinos $\tilde{\nu}$, charged sleptons $\tilde{l}$, and up- and down-type squarks $\tilde{u}$ and $\widetilde{d})$, spin-1/2 gluinos $(\tilde{g})$, spin-1/2 mixtures of neutral Higgsinos $\left(\widetilde{H}_{1-2}^{0}\right)$, the $B$-ino $(\widetilde{B})$, and the neutral $W$-ino $\left(\widetilde{W}^{3}\right)$, collectively called neutralinos $\left(\chi_{1-4}^{0}\right)$, and spin-1/2 mixtures of charged Higgsinos $\left(\widetilde{H}^{ \pm}\right)$and charged $W$-inos $\left(\widetilde{W}^{ \pm}\right)$, collectively called charginos $\left(\chi_{1,2}^{ \pm}\right)$. In addition, the Higgs sector of the MSSM contains two doublets (up and down types, which give mass to the up- and downtype fermions, respectively), whose vacuum expectations $v_{u}$ and $v_{d}$ are parametrized in terms of $v=\sqrt{v_{u}^{2}+v_{d}^{2}}$ and $\tan \beta$ $=v_{u} / v_{d}$. Together with the $\mathrm{SU}(2)_{L}$ and $\mathrm{U}(1)_{Y}$ couplings $g$ and $g^{\prime}$, respectively, $v$ is determined from $\alpha, M_{Z}$, and $G_{\mu}$, the Fermi constant extracted from the muon lifetime, while $\tan \beta$ remains a free parameter. The MSSM also introduces a coupling between the two Higgs doublets characterized by the dimensionful parameter $\mu$. The complete set Feynman rules for the MSSM, which take into account SUSY breaking and particle mixing, are given in Ref. [13].

Degeneracy between SM particles and their superpartners is lifted by the SUSY-breaking Lagrangian, which depends in general on 105 additional parameters. These include the SUSY-breaking Higgs mass parameters, the electroweak gaugino masses $M_{1,2}$, the gluino mass $M_{\tilde{g}}$, the left- (right-) handed sfermion mass parameters $M_{\widetilde{f}_{L}}^{2}\left(M_{\tilde{f}_{R}}^{2}\right)$, and left-right mixing terms $M_{\tilde{f}_{L R}}^{2}$ which mix $\tilde{f}_{L}$ and $\tilde{f}_{R}$ into mass eigenstates $\widetilde{f}_{1,2}$. In our analysis, we take the sfermion mass matrices to be diagonal in flavor space to avoid large flavorchanging neutral currents. We also set all $C P$-violating phases to zero. One expects the magnitude of the SUSYbreaking parameters to lie somewhere between the weak scale and $\sim 1 \mathrm{TeV}$. Significantly larger values can reintroduce the hierarchy problem.

Theoretical models for SUSY-breaking mediation provide relations among this large set of soft SUSY-breaking parameters, generally resulting in only a few independent parameters at the SUSY-breaking or GUT scale [14]. Evolution of the soft parameters down to the weak scale introduces flavor and species dependence into the superpartner spectrum due to the presence of Yukawa and gauge couplings in the renormalization group (RG) equations. According to the modelindependent analysis of Ref. [9], however, generic features of this spectrum implied by typical SUSY-breaking models and RG evolution may conflict with the combined constraints of low-energy charged current data, $M_{W}$, and the muon anomalous magnetic moment unless one allows for non-conservation of $P_{R}$. In light of this situation, we adopt here a similar model-independent approach and do not impose any specific relations among SUSY-breaking parameters. To our knowledge, no other model-independent analysis of MSSM corrections to PV observables has appeared in the literature, nor have the complete set of corrections to low-energy PV observables been computed previously (see, e.g. Ref. [15] for a study within minimal supergravity and gauge mediated models of SUSY breaking).

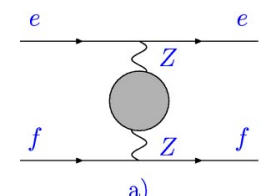

a)
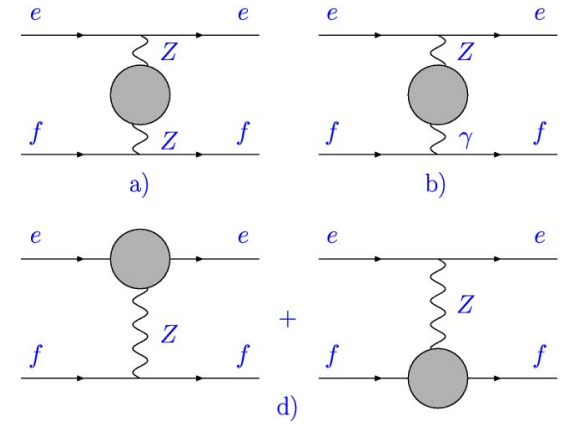

b)

d)
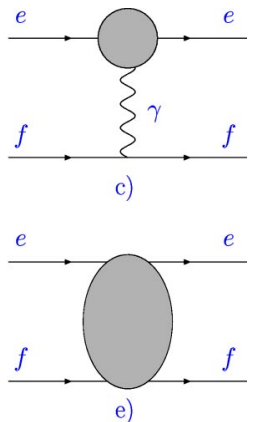

FIG. 1. Types of radiative corrections to parity-violating electron scattering: (a) $Z$ boson self-energy, (b) $Z-\gamma$ mixing, (c) electron anapole moment contributions, (d) vertex corrections, and (e) box graphs. External leg corrections are not explicitly shown.

\section{RADIATIVE CORRECTIONS TO $Q_{W}^{f}$}

With higher-order corrections included, the weak charge of a fermion $f$ can be written as

$$
Q_{W}^{f}=\rho_{P V}\left[2 I_{3}^{f}-4 Q_{f} \kappa_{P V} \sin ^{2} \theta_{W}\right]+\lambda_{f},
$$

where $I_{3}^{f}$ and $Q_{f}$ are, respectively, the weak isospin and the electric charge of the fermion $f$. The quantities $\rho_{P V}$ and $\kappa_{P V}$ are universal in that they do not depend on the fermion $f$ under consideration. The correction $\lambda_{f}$, on the other hand, does depend on the fermion species. At tree level, one has $\rho_{P V}=1=\kappa_{P V}$ and $\lambda_{f}=0$, while at one-loop order, these parameters are

$$
\begin{gathered}
\rho_{P V}=1+\delta \rho^{\mathrm{SM}}+\delta \rho^{\mathrm{SUSY}}, \\
\kappa_{P V}=1+\delta \kappa^{\mathrm{SM}}+\delta \kappa^{\mathrm{SUSY}}, \\
\lambda_{f}=\lambda_{f}^{\mathrm{SM}}+\lambda_{f}^{\mathrm{SUSY}},
\end{gathered}
$$

where the SUSY contributions to $\rho_{P V}, \kappa_{P V}$, and $\lambda_{f}$ are denoted in the above equation by the corresponding superscript. In general, the corrections $\delta \rho, \delta \kappa$, etc. depend on $q^{2}$, and in particular, the $q^{2}$ dependence of $\kappa_{P V}$ defines the scaledependence of the weak mixing angle: $\sin ^{2} \theta_{W}^{e f f}\left(q^{2}\right)$ $=\kappa_{P V}\left(q^{2}\right) \sin ^{2} \theta_{W}$, with $\sin ^{2} \theta_{W}$ being evaluated at some reference scale $q_{0}^{2}$ (usually $q_{0}^{2}=M_{Z}^{2}$ ).

The precise definitions of $\sin ^{2} \theta_{W}, \kappa_{P V}\left(q^{2}\right)$, etc. depend on one's choice of renormalization scheme. We evaluate the SUSY contributions using the modified dimensional reduction renormalization scheme $(\overline{\mathrm{DR}})[16]$ and denote all quantities evaluated in this scheme by a hat. In $\overline{\mathrm{DR}}$, all momenta are extended to $d=4-2 \epsilon$ dimensions, while the Dirac algebra remains four dimensional as required by SUSY invariance. The relevant classes of Feynman diagrams are shown in Fig. 1. Note that all gauge boson self-energies contribute only to $\rho_{P V}$ and $\kappa_{P V}$ while all non-universal box diagrams as well as vertex and external leg corrections are combined in $\lambda_{f}$. The counterterms for the effective PVES Lagrangian in Eq. (1) in the $\overline{\mathrm{DR}}$ scheme are given in Appendix A. 
The $Z$ boson self-energy contribution (Fig. 1a) simply rescales the leading order amplitude. Its effect is naturally combined with the counterterm $\delta \hat{G}_{\mu}$ from Eq. (A2) into $\rho_{P V}$ :

$$
\rho_{P V}=1+\frac{\delta \hat{G}_{\mu}}{G_{\mu}}+\frac{\hat{\Pi}_{Z Z}(0)}{M_{Z}^{2}}=1-\frac{\hat{\Pi}_{W W}(0)}{M_{W}^{2}}+\frac{\hat{\Pi}_{Z Z}(0)}{M_{Z}^{2}}-\hat{\delta}_{V B}^{\mu},
$$

where the $Z$ boson self-energy is evaluated at $q^{2}=0$. This is an appropriate approximation in this case because the momentum transfer in $e e$ and $e p$ scattering will be much smaller than the masses of the particles that appear in the loop graphs. The error is of the order $\left|q^{2}\right| / M_{Z}^{2} \sim 10^{-6}$, which is negligible. The quantity $\hat{\delta}_{V B}^{\mu}$ denotes the sum of electroweak vertex, external leg, and box graph corrections to the muon decay amplitude, which must be subtracted when the neutral current (NC) amplitudes are normalized to $G_{\mu}$.

The graphs Fig. 1(b),1(c) contribute to $\kappa_{P V}$ in Eq. (2). The expression is

$$
\kappa_{P V}=1+\frac{\hat{c}}{\hat{s}} \frac{\Pi_{Z \gamma}\left(q^{2}\right)}{q^{2}}+4 \hat{c}^{2} F_{A}^{e}\left(q^{2}\right)+\frac{\delta \hat{s}^{2}}{\hat{s}^{2}},
$$

where $F_{A}^{e}\left(q^{2}\right)$ is the parity-violating electron-photon form factor, which-at $q^{2}=0$-is known as the anapole moment of the electron [see Eq. (12)]. ${ }^{3}$ It should be noted that in the MSSM one has $\Pi_{Z \gamma}^{\operatorname{SUSY}}\left(q^{2}\right) \sim q^{2}$, so there is no singularity at $q^{2}=0$ in the above equation [17]. The SM contribution contains a singularity that is canceled by a corresponding singularity in the anapole moment contribution (Fig. 1c). Since in the following we consider only the new physics contributions, this issue is irrelevant (a complete treatment of the SM contributions is given in Refs. $[6,10,18])$. The shift $\delta \hat{s}^{2}$ in $\hat{s}^{2}=1-\hat{c}^{2} \equiv \sin ^{2} \hat{\theta}_{W}\left(M_{Z}^{2}\right)$ arises from its definition in terms of $\alpha, G_{\mu}$, and $M_{Z}$ :

$$
\begin{aligned}
\hat{s}^{2} \hat{c}^{2}= & \frac{\pi \alpha}{\sqrt{2} G_{\mu} M_{Z}^{2}(1-\Delta \hat{r})}, \\
\Delta \hat{r}= & \Pi_{\gamma \gamma}^{\prime}(0)+2 \frac{\hat{s}}{\hat{c}} \frac{\hat{\Pi}_{Z \gamma}(0)}{M_{Z}^{2}}-\frac{\Pi_{Z Z}\left(M_{Z}^{2}\right)}{M_{Z}^{2}} \\
& +\frac{\Pi_{W W}(0)}{M_{W}^{2}}+\hat{\delta}_{V B}^{\mu},
\end{aligned}
$$

where $\hat{\Pi}_{\gamma \gamma}^{\prime}\left(q^{2}\right) \equiv \hat{\Pi}_{\gamma \gamma} / q^{2}$. Writing $\Delta \hat{r}=\Delta \hat{r}^{\mathrm{SM}}+\Delta \hat{r}^{\mathrm{SUSY}}$ one has

$$
\frac{\delta \hat{s}_{\text {SUSY }}^{2}}{\hat{s}^{2}}=\frac{\hat{c}^{2}}{\hat{c}^{2}-\hat{s}^{2}} \Delta \hat{r}^{\text {SUSY }} .
$$

\footnotetext{
${ }^{3}$ Note that our definition of $\kappa_{P V}$, Eq. (5), includes the anapole form factor of the electron $F_{A}^{e}\left(q^{2}\right)$, which may be absent in other definitions appearing in literature (see, e.g. Ref. [6]).
}

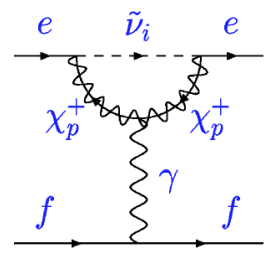

(a)

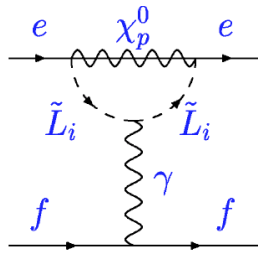

(b)
FIG. 2. Electron anapole moment contributions to the parityviolating electron-fermion scattering amplitude.

In computing the SUSY corrections to the weak charges one must decide which value for $\hat{s}^{2}$ to use. Since $\delta \hat{s}_{\text {SUSY }}^{2}$ has already been absorbed into $\kappa_{P V}$ one must determine $\hat{s}^{2}$ from Eq. (6) using the SM radiative corrections only. The corresponding value extracted using only $\alpha, G_{\mu}$, and $M_{Z}$ is [12]:

$$
\hat{s}^{2}=0.23120 \pm 0.00018
$$

In order to incorporate constraints from existing precision data (see Sec. V), it is useful to introduce the oblique parameters $S, T$, and $U[19]$ :

$$
\begin{aligned}
S= & \frac{4 \hat{s}^{2} \hat{c}^{2}}{\hat{\alpha} M_{Z}^{2}} \operatorname{Re}\left\{\hat{\Pi}_{Z Z}(0)-\hat{\Pi}_{Z Z}\left(M_{Z}^{2}\right)+\frac{\hat{c}^{2}-\hat{s}^{2}}{\hat{c} \hat{s}}\left[\hat{\Pi}_{Z \gamma}\left(M_{Z}^{2}\right)\right.\right. \\
& \left.\left.-\hat{\Pi}_{Z \gamma}(0)\right]+\hat{\Pi}_{\gamma \gamma}\left(M_{Z}^{2}\right)\right\}^{\text {New }}, \\
T= & \frac{1}{\hat{\alpha} M_{W}^{2}}\left\{\hat{c}^{2}\left(\Pi_{Z Z}(0)+\frac{2 \hat{s}}{\hat{c}} \hat{\Pi}_{Z \gamma}(0)\right)-\hat{\Pi}_{W W}(0)\right\}^{\text {New }}, \\
U= & \frac{4 \hat{s}^{2}}{\hat{\alpha}}\left\{\frac{\Pi_{W W}(0)-\Pi_{W W}\left(M_{W}^{2}\right)}{M_{W}^{2}}+\hat{c}^{2} \frac{\Pi_{Z Z}\left(M_{Z}^{2}\right)-\Pi_{Z Z}(0)}{M_{Z}^{2}}\right. \\
& \left.+2 \hat{c} \hat{s} \frac{\Pi_{Z \gamma}\left(M_{Z}^{2}\right)-\hat{\Pi}_{Z \gamma}(0)}{M_{Z}^{2}}+\hat{s}^{2} \frac{\Pi_{\gamma \gamma}\left(M_{Z}^{2}\right)}{M_{Z}^{2}}\right\}^{\text {New }},
\end{aligned}
$$

where the superscript "New" indicates that only the new physics contributions to the self-energies are included. Contributions to gauge-boson self-energies can be expressed entirely in terms of the oblique parameters $S, T$, and $U$ in the limit that $M_{\mathrm{NEW}} \gg M_{Z}$. However, since present collider limits allow for fairly light superpartners, we do not work in this limit. Consequently, the corrections arising from the photon self-energy $\left(\Pi_{\gamma \gamma}\right)$ and $\gamma-Z$ mixing tensor $\left(\Pi_{Z \gamma}\right)$ contain a residual $q^{2}$ dependence not embodied by the oblique parameters. Expressing $\rho_{P V}$ and $\kappa_{P V}$ in terms of $S, T$, and $U$ we obtain

$$
\delta \rho^{\mathrm{SUSY}}=\hat{\alpha} T-\hat{\delta}_{V B}^{\mu},
$$




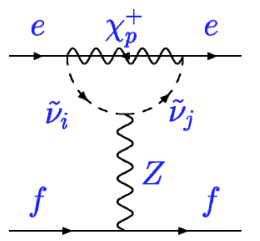

(a)

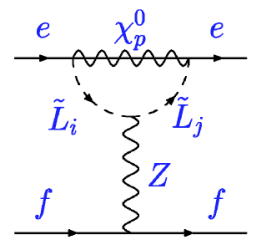

(b)

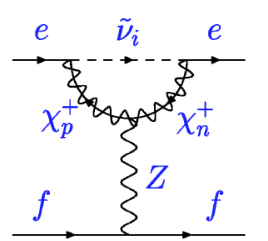

(c)

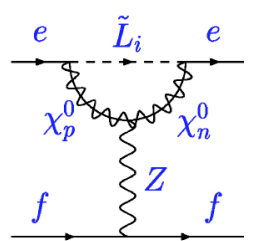

(d)

$$
\begin{aligned}
\delta \kappa^{\mathrm{SUSY}}= & \left(\frac{\hat{c}^{2}}{\hat{c}^{2}-\hat{s}^{2}}\right)\left(\frac{\hat{\alpha}}{4 \hat{s}^{2} \hat{c}^{2}} S-\hat{\alpha} T+\hat{\delta}_{V B}^{\mu}\right)+\frac{\hat{c}}{\hat{s}}\left[\frac{\hat{\Pi}_{Z \gamma}\left(q^{2}\right)}{q^{2}}\right. \\
& \left.-\frac{\hat{\Pi}_{Z \gamma}\left(M_{Z}^{2}\right)}{M_{Z}^{2}}\right]^{\mathrm{SUSY}}+\left(\frac{\hat{c}^{2}}{\hat{c}^{2}-\hat{s}^{2}}\right)\left[-\frac{\hat{\Pi}_{\gamma \gamma}\left(M_{Z}^{2}\right)}{M_{Z}^{2}}\right. \\
& \left.+\frac{\Delta \hat{\alpha}}{\alpha}\right]^{\mathrm{SUSY}}+4 \hat{c}^{2} F_{A}^{e}\left(q^{2}\right)^{\mathrm{SUSY}},
\end{aligned}
$$

where $\Delta \hat{\alpha}$ is the SUSY contribution to the difference between the fine structure constant and the electromagnetic coupling renormalized at $\mu=M_{Z}: \Delta \hat{\alpha}=\left[\hat{\alpha}\left(M_{Z}\right)-\alpha\right]^{\mathrm{SUSY}}$. As noted above, we take $q^{2} \rightarrow 0$ in our analysis.

The non-universal contribution to the weak charge is determined by the sum of the renormalized vertex corrections $\hat{V}_{V, A}^{f}$ in Fig. 1(d) [see Eq. (A6)] and the box graphs $\hat{\delta}_{\text {Box }}^{e f}$ in Fig. 1(e) [see Eq. (C15)]:

$$
\hat{\lambda}_{f}=g_{V}^{f} \hat{V}_{A}^{e}+g_{A}^{e} \hat{V}_{V}^{f}+\hat{\delta}_{\mathrm{Box}}^{e f},
$$

where $g_{V, A}^{f}$ are given in Eq. (A3).

Finally, we note that by vector current conservation, $\delta Q_{W}^{p}$ can be computed directly from the shifts in the up- and down-quark weak charges: $\delta Q_{W}^{p}=2 \delta Q_{W}^{u}+\delta Q_{W}^{d}$. The analogous relation in the SM is modified by non-perturbative strong interactions in the $Z \gamma$ box graph [6]. The latter arise because the loop contains a massless particle, rendering the corresponding loop integral sensitive to both low and high momentum scales. In contrast, the SUSY radiative corrections are dominated by large loop momenta, and nonperturbative QCD corrections are suppressed by $\left(\Lambda_{\mathrm{QCD}} / M_{\mathrm{SUSY}}\right)^{2} \ll 1$.

\section{One-loop SUSY Feynman diagrams}

Here, we present the SUSY one-loop diagrams that are particular to PVES. Such diagrams correspond to the generic corrections shown in Figs. 1(c), 1(d), and 1(e). Contributions corresponding to Figs. 1(a) and 1(b) are universal, and the relevant diagrams - together with the external leg corrections
FIG. 3. MSSM radiative corrections to the electron neutral current vertex. Radiative corrections to the down quark vertex are obtained by replacing charged leptons (sleptons, $\widetilde{L}_{i}$ ) with down type quarks (squarks) and sneutrinos with up type squarks.

for all fermions-are given in Ref. [11]. In addition, some simplifications occur in the analysis for PVES that do not arise in general. In the case of charged current observables, for example, gluino loops can generate substantial corrections $[9,11]$. In contrast, gluinos decouple entirely from the one-loop MSSM corrections to semi-leptonic neutral current PV observables. The proof of this statement is given in Appendix B. In addition, the MSSM Higgs contributions to vertex, external leg, and box graph corrections are negligible due to the small, first- and second-generation Yukawa couplings. The light Higgs contribution to gauge boson propagators has already been included via the oblique parameters, while the effects of other MSSM Higgs bosons are sufficiently small to be neglected [20]. Therefore, we do not discuss gluino and Higgs contributions in the following.

Anapole moment corrections, corresponding to Fig. 1(c). In the presence of parity-violating interactions, higher-order contributions can generate the photon-fermion coupling of the form (see, e.g. Ref. [21]):

$$
i \mathcal{M}_{\gamma-f}^{P V}=-i e \frac{F_{A}^{f}\left(q^{2}\right)}{M_{Z}^{2}} \bar{f}\left(q^{2} \gamma^{\mu}-\not q^{\mu}\right) \gamma_{5} f \varepsilon_{\mu},
$$

where $f$ is a fermion spinor, $\varepsilon_{\mu}$ is the photon polarization, and $F_{A}^{f}\left(q^{2}\right)$ is the anapole moment form factor.

The quantity $F_{A}^{f}\left(q^{2}\right)$ is, in general, gauge dependent. This dependence cancels after the anapole moment contribution is combined with other one-loop corrections to the given scattering process [21]. The Feynman diagrams that contribute to $F_{A}^{e}\left(q^{2}\right)^{\text {SUSY }}$ are shown in Fig. 2, and the analytical expressions are presented in Appendix C.

Vertex corrections, corresponding to Fig. 1(d). The relevant diagrams are shown in Figs. 3 and 4. The diagrams in Fig. 3 cover $e e, e d$, and $e u$ scattering when the radiative correction is for the projectile side. When the radiative correction is to be applied to the target side, the diagrams in Fig. 3 can also be used for $e e$ and $e d$ scattering. In this case $f$ $=e$ is the projectile. To obtain the corrections to the down quark vertex, the electron can simply be replaced with the down quark. The diagrams in Fig. 4 show the radiative corrections to the target side when the incoming electron inter-

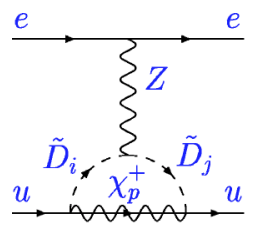

(a)

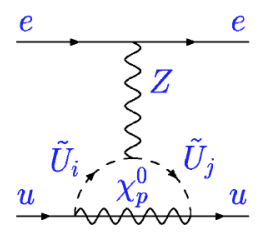

(b)

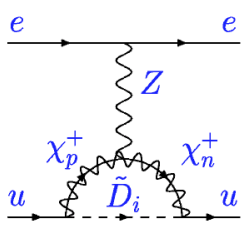

(c)

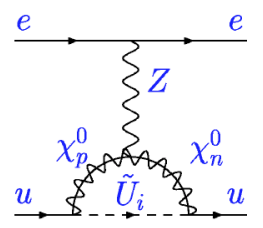

(d)
FIG. 4. MSSM radiative corrections to the up quark neutral current vertex. Here, $\widetilde{U}_{i}\left(\widetilde{D}_{i}\right)$ denotes up(down)-type squark. 


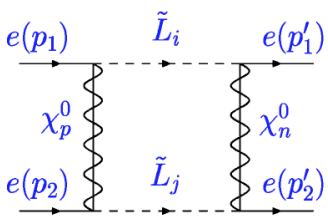

a)

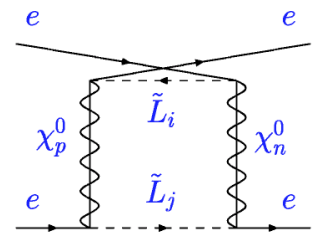

b)

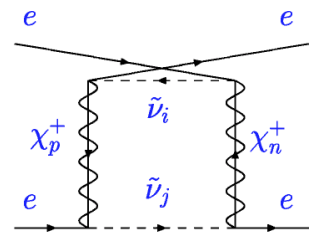

c)
FIG. 5. MSSM box graphs that contribute to the electron-electron scattering amplitude. Here, $p_{i}\left(p_{i}^{\prime}\right), i=1,2$, is the momentum of the initial (final) state fermion. Radiative corrections to the electron-down quark scattering are trivially obtained by replacing the target with the down quark. acts with the up quark inside the proton. The explicit expressions for the vertex corrections can be found in Appendix C.

Box corrections, corresponding to Fig. 1(e). These graphs generate $\hat{\delta}_{\text {Box }}^{e f}$ in Eq. (11). The relevant diagrams are shown in Fig. 5 and Fig. 6. The explicit expressions are given in Appendix C.

\section{IV. $R$-PARITY VIOLATING CONTRIBUTIONS TO $Q_{W}^{f}$}

When $R$ parity is not conserved, new tree-level contributions to $Q_{W}^{e, p}$ appear. The latter are generated by the $(B$ $-L)$-violating superpotential:

$$
\begin{aligned}
W_{R P V}= & \frac{1}{2} \lambda_{i j k} L_{i} L_{j} \bar{E}_{k}+\lambda_{i j k}^{\prime} L_{i} Q_{j} \bar{D}_{k}+\frac{1}{2} \lambda_{i j k}^{\prime \prime} \bar{U}_{i} \bar{D}_{j} \bar{D}_{k} \\
& +\mu_{i}^{\prime} L_{i} H_{u},
\end{aligned}
$$

where $L_{i}$ and $Q_{i}$ denote lepton and quark $\mathrm{SU}(2)_{L}$ doublet superfields, $E_{i}, U_{i}$, and $D_{i}$ are singlet superfields and the $\lambda_{i j k}$, etc. are a priori unknown couplings. In order to avoid unacceptably large contributions to the proton decay rate, we set the $\Delta B \neq 0$ couplings $\lambda_{i j k}^{\prime \prime}$ to zero. For simplicity, we also neglect the last term in Eq. (13). The purely leptonic terms $\left(\lambda_{12 k}\right)$ contribute to the electron scattering amplitudes via the normalization of NC amplitudes to $G_{\mu}$ and through the definition of $\hat{s}^{2}$ [22]. The remaining semileptonic, $\Delta L= \pm 1$ interactions $\left(\lambda_{i j k}^{\prime}\right)$ give direct contributions to the $e q$ scattering amplitudes. The latter may be obtained computing the Feynman amplitudes in Figs. 7(b),7(c) and preforming a Fierz reordering. In this manner one obtains the following effective four-fermion Lagrangian:

$$
\begin{aligned}
\mathcal{L}_{R P V}^{E F F}= & -\frac{\left|\lambda_{1 k 1}^{\prime}\right|^{2}}{2 M_{\tilde{q}_{L}^{k}}^{2}} \bar{d}_{R} \gamma^{\mu} d_{R} \bar{e}_{L} \gamma_{\mu} e_{L}+\frac{\left|\lambda_{11 k}^{\prime}\right|^{2}}{2 M_{\tilde{d}_{R}^{k}}^{2}} \bar{u}_{L} \gamma^{\mu} u_{L} \bar{e}_{L} \gamma_{\mu} e_{L} \\
& -\frac{\left|\lambda_{12 k}\right|^{2}}{2 M_{\tilde{e}_{R}^{k}}^{2}}\left[\bar{\nu}_{\mu L} \gamma^{\mu} \mu_{L} \bar{e}_{L} \gamma_{\mu} \nu_{e L}+\text { H.c. }\right]
\end{aligned}
$$

where we have taken $\left|q^{2}\right| \ll M_{\tilde{f}}^{2}$ and have retained only the terms relevant for the PVES scattering. Note the absence from Eq. (14) of the parity-violating contact four-electron interaction. It is straightforward to show that the superpotential in Eq. (13) can only produce parity-conserving contact interactions between identical leptons.

Contributions from $P_{R}$-violating interactions to low energy observables can be parametrized in terms of the following quantities:

$$
\Delta_{i j k}(\widetilde{f})=\frac{\left|\lambda_{i j k}\right|^{2}}{4 \sqrt{2} G_{\mu} M_{\tilde{f}}^{2}} \geqslant 0,
$$

with a similar definition for the primed quantities. In terms of $\Delta_{i j k}$, etc., one obtains for the relative shifts in the weak charges [22]:

$$
\begin{aligned}
\frac{\delta Q_{W}^{e}}{Q_{W}^{e}} \approx & -\left[1+\left(\frac{4}{1-4 \sin ^{2} \theta_{W}}\right) \lambda_{x}\right] \Delta_{12 k}\left(\tilde{e}_{R}^{k}\right) \\
\frac{\delta Q_{W}^{p}}{Q_{W}^{p}} \approx & \left(\frac{2}{1-4 \sin ^{2} \theta_{W}}\right)\left[-2 \lambda_{x} \Delta_{12 k}\left(\tilde{e}_{R}^{k}\right)+2 \Delta_{11 k}^{\prime}\left(\widetilde{d}_{R}^{k}\right)\right. \\
& \left.-\Delta_{1 k 1}^{\prime}\left(\tilde{q}_{L}^{k}\right)\right]-\Delta_{12 k}\left(\tilde{e}_{R}^{k}\right), \\
\lambda_{x}= & \frac{\hat{s}^{2}\left(1-\hat{s}^{2}\right)}{1-2 \hat{s}^{2}} \frac{1}{1-\Delta \hat{r}^{\mathrm{SM}}} \approx 0.35 .
\end{aligned}
$$

As discussed in Sec. V the quantities $\Delta_{i j k}$, etc. are constrained from other precision data. Since they are nonnegative, Eq. (16) indicates that the relative shift in $Q_{W}^{e}$ is negative semidefinite. On the other hand, the relative shift in $Q_{W}^{p}$ can have either sign depending on the relative magnitudes of $\Delta_{12 k}, \Delta_{11 k}^{\prime}$, and $\Delta_{1 k 1}^{\prime}$.

\section{ANALYSIS OF THE SUSY CONTRIBUTIONS TO THE WEAK CHARGES}

In order to evaluate the potential size of SUSY loop corrections, a set of about 3000 different combinations of SUSY-breaking parameters was generated, chosen randomly from a flat distribution in the soft SUSY mass parameters (independent for each generation) and in $\ln \tan \beta$. The

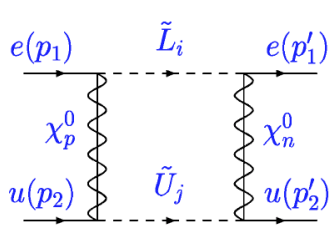

a)

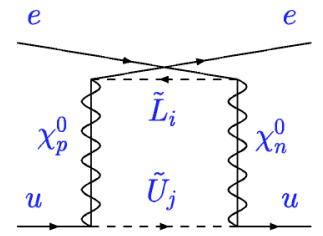

b)

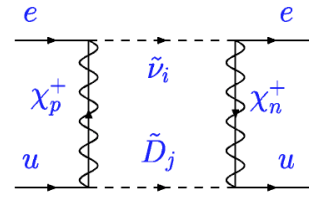

c)
FIG. 6. MSSM box graphs that contribute to the electron-up quark scattering. The meaning of momentum labels is the same as in Fig. 5. 


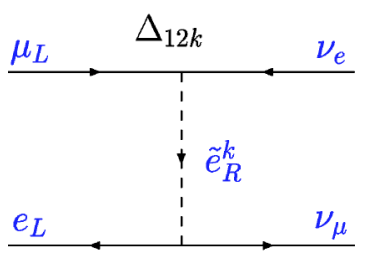

a)

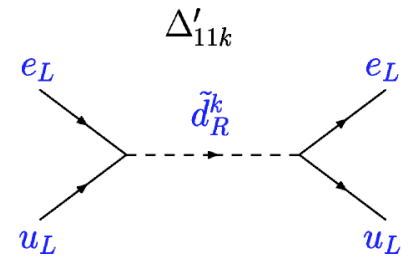

b)

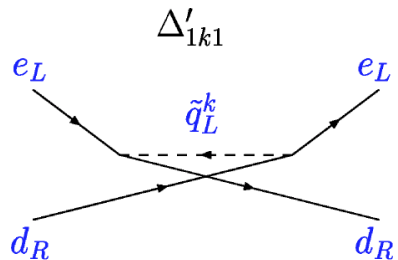

c)
FIG. $\quad 7 . \quad$ Tree-level $P_{R}$-violating contributions to the muon decay [plot (a)], the eu scattering amplitude [plot (b)], and the ed scattering amplitude [plot (c)]. The quantities $\Delta_{i j k}$, etc., are defined in Eq. (15). former were bounded below by present collider limits and bounded above by $1000 \mathrm{GeV}$, corresponding to the $\mathcal{O}(\mathrm{TeV})$ naturalness limit. Also, $\tan \beta$ was restricted to lie in the range $1.4<\tan \beta<60$. These limits follow from the requirement that the third generation quark Yukawa couplings remain perturbative (small) up to the grand unified theory (GUT) scale. Left-right mixing among sfermions was allowed. In order to avoid unacceptably large flavor-changing neutral currents, no intergenerational sfermion mixing was permitted. The ranges over which the soft SUSY breaking parameters and $\tan \beta$ were scanned are shown in Table I.

For each combination of parameters, we computed superpartner masses and mixing angles, which we then used as inputs for computing the radiative corrections. Only the parameters generating SUSY contributions to the muon anomalous magnetic moment consistent with the latest results [23] were considered. We also separately evaluated the corresponding contributions to the oblique parameters. The latter are tightly constrained from precision electroweak data. We rule out any parameter combination leading to values of $S$ and $T$ lying outside the present $95 \%$ confidence limit contour for these quantities. We note that this procedure is not entirely self-consistent, since we have not evaluated nonuniversal MSSM corrections to other precision electroweak observables before extracting oblique parameter constraints. As noted in Ref. [15], where MSSM corrections to Z-pole observables were evaluated using different models for SUSY-breaking mediation, non-universal effects can be as large as oblique corrections. Nevertheless, we expect our procedure to yield a reasonable estimate of the oblique parameter constraints. Since $S$ and $T$ do not dominate the lowenergy SUSY corrections (see below), our results depend only gently on the precise allowed ranges for these parameters.

In Fig. 8 we plot the shift in the weak charge of the proton, $\delta Q_{W}^{p}=2 \delta Q_{W}^{u}+\delta Q_{W}^{d}$, versus the corresponding shift in the electron's weak charge, $\delta Q_{W}^{e}$, normalized to the respective SM values. The corrections in the MSSM (with $P_{R}$

TABLE I. Ranges of SUSY parameters scanned. Here, $\tilde{M}$ denotes any of $|\mu|, M_{1,2}$, or the diagonal sfermion mass parameters $M_{\tilde{f}_{L, R}}^{i}$. The $\mu$ parameter and $M_{1,2}$ can take either sign. The generation index $i$ runs from 1 to 3 .

\begin{tabular}{ccc}
\hline \hline Parameter & Min & Max \\
\hline $\tan \beta$ & 1.4 & 60 \\
$\tilde{M}$ & $50 \mathrm{GeV}$ & $1000 \mathrm{GeV}$ \\
$\left(M_{\tilde{f}}^{2}\right)_{L R}^{i}$ & $-10^{6} \mathrm{GeV}^{2}$ & $10^{6} \mathrm{GeV}^{2}$ \\
\hline \hline
\end{tabular}

conserved) can be as large as $\sim 4 \%\left(Q_{W}^{p}\right)$ and $\sim 8 \%$ $\left(Q_{W}^{e}\right)$-roughly the size of the proposed experimental errors for the two PVES measurements. Generally speaking, the magnitudes of $\delta Q_{W}^{e, p}$ slowly increase with $\tan \beta$ and decrease as SUSY mass parameters are increased. The largest effects occur when at least one superpartner is relatively light. An exception occurs in the presence of significant mass splitting between sfermions, which may lead to sizable contributions. However, such weak isospin-breaking effects also increase the magnitude of $T$, so their impact is bounded by oblique parameter constraints. This consideration has been implemented in arriving at Fig. 8.

The effects of sfermion left-right mixing were studied separately. We observe that the presence or the absence of the mixing affects the distribution of points, but does not significantly change the range of possible corrections. For the situation of no left-right mixing, the points are more strongly clustered near the origin. Thus, while corrections of the order of several percent are possible in either case, large effects are more likely in the presence of left-right mixing.

The shifts $\delta Q_{W}^{e, p}$ are dominated by $\delta \kappa^{\mathrm{SUSY}}$. This feature is illustrated for $Q_{W}^{e}$ in Fig. 9 where the soft SUSY breaking parameters are chosen such that the total SUSY correction to $Q_{W}^{e}$ is about $4 \%$, about a half of its maximum value. For $Q_{W}^{p}$ the situation is similar. We observe that non-universal corrections involving vertex corrections and wave function renormalization experience significant cancellations. In addition,

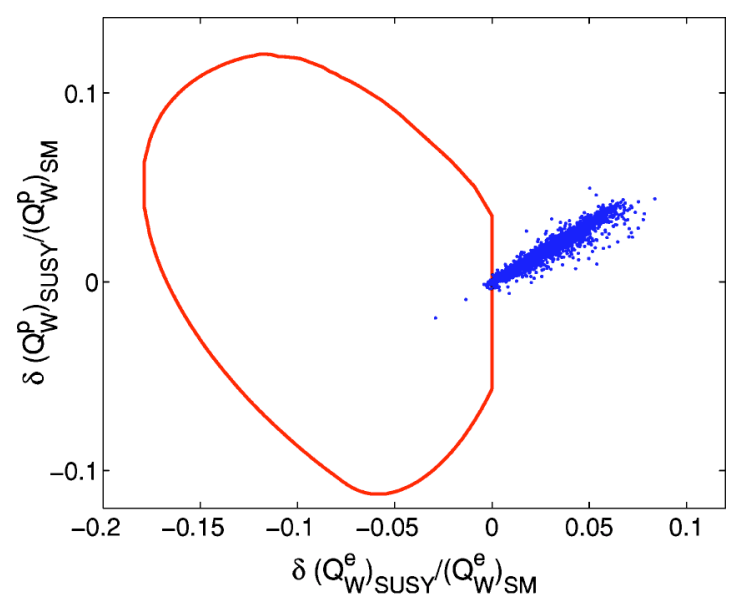

FIG. 8. Relative shifts in electron and proton weak charges due to SUSY effects. Dots indicate SUSY loop corrections for $\sim 3000$ randomly generated SUSY-breaking parameters. The interior of the truncated elliptical region gives possible shifts due to $P_{R}$ nonconserving SUSY interactions (95\% confidence). 


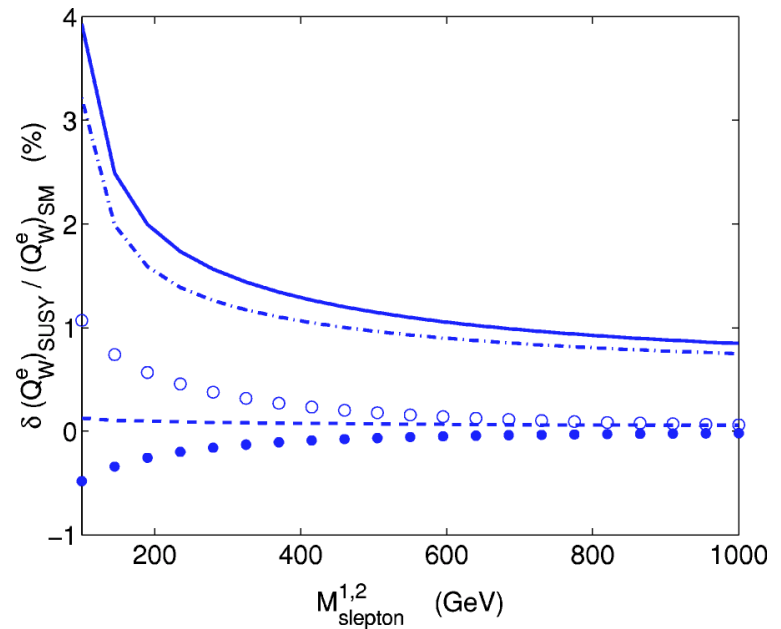

FIG. 9. Various contributions to $\delta Q_{W}^{e} / Q_{W}^{e}$ : total from SUSY loops (solid line), from $\delta \rho^{\mathrm{SUSY}}$ (dashed line), from $\delta \kappa^{\mathrm{SUSY}}$ (dashdotted line), from the vertex corrections (dotted line), and from the box graphs (open circles). The $x$ axis gives the lepton superpartner mass, chosen to be the same for both left- and right-handed first and second generation sleptons. For this graph, $\tan \beta=10$, the gaugino soft mass parameters are $2 M_{1}=M_{2}=\mu=200 \mathrm{GeV}$, and masses of the third generation sleptons and of all squarks are $1000 \mathrm{GeV}$. For this case, $\delta Q_{W}^{e} / Q_{W}^{e}$ is about half of its maximum possible value. The total relative correction is clearly dominated by $\delta \kappa^{\mathrm{SUSY}}$.

corrections to $Q_{W}^{e, p}$ due to shifts in the $\rho_{P V}$ parameter are suppressed by $1-4 \hat{s}^{2}$.

We find that $\delta \kappa^{\text {SUSY }}$ is nearly always negative, corresponding to a reduction in the value of $\sin ^{2} \theta_{W}^{e f f}\left(q^{2}\right)$ $=\kappa_{P V}\left(q^{2}\right) \sin ^{2} \theta_{W}$ for the parity-violating electron scattering experiments [see Eq. (2)]. In this case, the degree of cancellation between $2 I_{f}^{3}$ and $Q_{f}$ terms in Eq. (2) is reduced, yielding an increased magnitude of $Q_{W}^{f}$. Since this effect is identical for both $Q_{W}^{e}$ and $Q_{W}^{p}$, the dominant effect of $\delta \kappa$ produces a linear correlation between the two weak charges. Some scatter around this line arises from non-universal effects in $\hat{\lambda}_{f}$ (see Fig. 8).

As illustrated in Fig. 10, within $\delta \kappa^{\text {SUSY }}$ itself, contributions from the various terms in Eq. (10) have comparable importance, with some degree of cancellation occurring between the effects of $S$ and $T$. Thus, the oblique parameter approximation gives a rather poor description of the MSSM effects on the weak charges. In particular the quantity $\hat{\delta}_{V B}^{\mu}$ in Eq. (10) makes a significant contribution to $\delta \kappa^{\mathrm{SUSY}}$.

As evident from Fig. 8, the relative sign of the corrections to both $Q_{W}^{p}$ and $Q_{W}^{e}$-normalized to the corresponding SM values -is nearly always the same and nearly always positive. Since $Q_{W}^{p}>0\left(Q_{W}^{e}<0\right)$ in the SM, SUSY loop corrections give $\delta Q_{W}^{p}>0\left(\delta Q_{W}^{e}<0\right)$. This correlation is significant, since the effects of other new physics scenarios can display different signatures. For example, for the general class of theories based on $E_{6}$ gauge group, with neutral gauge bosons having mass $\lesssim 1000 \mathrm{GeV}$, the effects on $Q_{W}^{p}$ and $Q_{W}^{e}$ also correlate, but $\delta Q_{W}^{e, p} / Q_{W}^{e, p}$ can have either sign in this case $[6,24]$. In contrast, leptoquark interactions would

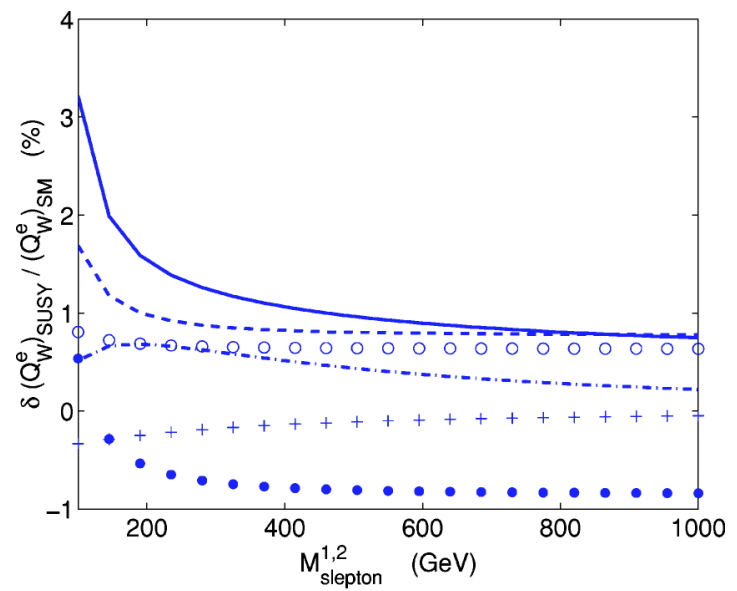

FIG. 10. Contributions to $\delta Q_{W}^{e} / Q_{W}^{e}$ from various corrections to $\delta \kappa^{\mathrm{SUSY}}$ [see Eq. (10)]: total from $\delta \kappa^{\mathrm{SUSY}}$ (solid line), $S$ parameter (dotted line), $T$ parameter (dashed line), $\hat{\delta}_{V B}^{\mu}$ (dash-dotted line), $Z-\gamma$ mixing and the photon self-energy (open circles), and the electron anapole moment (crosses). The soft SUSY parameters are the same as in Fig. 9.

not lead to discernible effects in $Q_{W}^{e}$ but could induce sizable shifts in $Q_{W}^{p}[6,24]$.

As a corollary, we also find that the relative importance of SUSY loop corrections to the weak charge of heavy nuclei probed with APV is suppressed. The shift in the nuclear weak charge is given by $\delta Q_{W}(Z, N)=(2 Z+N) \delta Q_{W}^{u}+(2 N$ $+Z) \delta Q_{W}^{d}$. Since the sign of $\delta Q_{W}^{f} / Q_{W}^{f}$ due to superpartner loops is nearly always the same, and since $Q_{W}^{u}>0$ and $Q_{W}^{d}$ $<0$ in the SM, a strong cancellation between $\delta Q_{W}^{u}$ and $\delta Q_{W}^{d}$ occurs in heavy nuclei. This cancellation implies that the magnitude of $\delta Q_{W}(Z, N) / Q_{W}(Z, N)$ is generally less than about $0.2 \%$ for cesium and is equally likely to have either sign. Since the presently quoted uncertainty for the cesium nuclear weak charge is about $0.6 \%$ [25], cesium APV does not substantially constrain the SUSY parameter space. Equally as important, the present agreement of $Q_{W}^{\mathrm{Cs}}$ with the SM prediction does not preclude significant shifts in $Q_{W}^{e, p}$ arising from SUSY. The situation is rather different, for example, in the $E_{6} Z^{\prime}$ scenario, where sizable shifts in $Q_{W}^{e, p}$ would also imply observable deviations of $Q_{W}^{\mathrm{Cs}}$ from the SM prediction.

The prospective "diagnostic power" of the two PVES measurements is further increased when one relaxes the assumption of $P_{R}$ conservation. Doing so leads to the tree-level corrections to the weak charges shown in Eq. (16). The quantities $\Delta_{i j k}$, etc. in Eqs. (15) and (16) are constrained from the existing precision data [22]. A summary of the existing constraints-including the latest theoretical inputs into the extraction of $Q_{W}^{\mathrm{Cs}}$ from experiment [25]—is given in Table II of Ref. [11], which we partially reproduce here in Table II. We list the $P_{R}$-violating contribution to four relevant precision observables: superallowed nuclear $\beta$ decay that constrains $\left|V_{u d}\right|$ [26], atomic PV measurements of the cesium weak charge $Q_{W}^{\mathrm{Cs}}$ [2], the $e / \mu$ ratio $R_{e / \mu}$ in $\pi_{l 2}$ decays [27], and a comparison of the Fermi constant $G_{\mu}$ with the appropriate combination of $\alpha, M_{Z}$, and $\sin ^{2} \theta_{W}$ [28]. The values of 
TABLE II. $P_{R^{-}}$violating contributions to $\delta\left|V_{u d}\right|^{2} /\left|V_{u d}\right|^{2}, \delta Q_{W}^{\mathrm{Cs}} / Q_{W}^{\mathrm{Cs}}, \delta R_{e / \mu}, \delta G_{\mu} / G_{\mu}, \delta Q_{W}^{p} / Q_{W}^{p}$, and $\delta Q_{W}^{e} / Q_{W}^{e}$. Columns give the coefficients of the various corrections from $\Delta_{i j k}^{\prime}$ and $\Delta_{12 k}$ to the different quantities. The last column gives the experimentally measured value of the corresponding quantity (for $Q_{W}^{p, e}$, only the proposed experimental uncertainties are shown).

\begin{tabular}{cccccc}
\hline \hline Quantity & $\Delta_{11 k}^{\prime}\left(\widetilde{d}_{R}^{k}\right)$ & $\Delta_{1 k 1}^{\prime}\left(\tilde{q}_{L}^{k}\right)$ & $\Delta_{12 k}\left(\tilde{e}_{R}^{k}\right)$ & $\Delta_{21 k}^{\prime}\left(\tilde{d}_{R}^{k}\right)$ & Value \\
\hline$\delta\left|V_{u d}\right|^{2} /\left|V_{u d}\right|^{2}$ & 2 & 0 & -2 & 0 & $-0.0029 \pm 0.0014$ \\
$\delta Q_{W}^{\mathrm{Cs}} / Q_{W}^{\mathrm{Cs}}$ & -4.82 & 5.41 & 0.05 & 0 & $-0.0040 \pm 0.0066$ \\
$\delta R_{e / \mu}$ & 2 & 0 & 0 & -2 & $-0.0042 \pm 0.0033$ \\
$\delta G_{\mu} / G_{\mu}$ & 0 & 0 & 1 & 0 & $0.00025 \pm 0.001875$ \\
$\delta Q_{W}^{p} / Q_{W}^{p}$ & 55.9 & -27.9 & -18.7 & 0 & \pm 0.040 \\
$\delta Q_{W}^{e} / Q_{W}^{e}$ & 0 & 0 & -29.8 & 0 & \pm 0.089 \\
\hline \hline
\end{tabular}

the experimental constraints on those quantities are given in the last column. We also list the $P_{R^{-} \text {-violating contributions to }}$ $\delta Q_{W}^{p} / Q_{W}^{p}$ and $\delta Q_{W}^{e} / Q_{W}^{e}$, along with proposed experimental uncertainties.

The $95 \%$ C.L. region allowed by this fit in the $\delta Q_{W}^{p} / Q_{W}^{p}$ vs $\delta Q_{W}^{e} / Q_{W}^{e}$ plane is shown by the closed curve in Fig. 8 . Note that the sign requirements $\Delta_{i j k}(\widetilde{f}), \Delta_{i j k}^{\prime}(\widetilde{f}) \geqslant 0[$ see Eq. (15)] truncate the initially elliptical curve to the shape shown in the figure. We observe that the prospective effects of $P_{R}$ non-conservation are quite distinct from SUSY loops. The value of $\delta Q_{W}^{e} / Q_{W}^{e}$ is never positive in contrast to the situation for SUSY loop effects, whereas $\delta Q_{W}^{p} / Q_{W}^{p}$ can have either sign. Note, however, that the area enclosed by the curve corresponding to $\delta Q_{W}^{p} / Q_{W}^{p} \geqslant 0$ is larger than the area corresponding to $\delta Q_{W}^{p} / Q_{W}^{p}<0$, implying that $\delta Q_{W}^{p} / Q_{W}^{p}$ is more likely to be positive. In addition, the magnitude of the $P_{R}$-violating effects can be roughly twice as large as the possible magnitude of SUSY loop effects for both $Q_{W}^{e, p}$. Thus, a comparison of results for the two parity-violating electron scattering experiments could help determine whether this extension of the MSSM is to be favored over other new physics scenarios (see also Ref. [6]).

\section{CONCLUSIONS}

A new generation of precise, PVES experiments are poised to probe a variety of scenarios for physics beyond the SM $[4-6,10]$. The sensitivity of these measurements to new physics is enhanced because the SM values for the electron and proton weak charges are suppressed and because theoretical uncertainties in the SM predictions are sufficiently small $[6,10]$. Here, we have studied the ability of these measurements to shed new light on supersymmetric extensions of the SM. We have observed that in a $P_{R}$-conserving version of the MSSM, the effects of SUSY loop corrections to the electron and proton weak charges are highly correlated and have the same relative sign (positive) compared to the SM prediction over nearly all the available MSSM parameter space. This correlation arises because the corrections are dominated by the SUSY loop contributions to $\sin ^{2} \theta_{W}^{e f f}\left(q^{2}\right)-\mathrm{a}$ result that would not have been obvious in the absence of an explicit calculation. Moreover, the appearance of this correlation does not result from the adoption of any model for
SUSY-breaking mediation, as we have undertaken a modelindependent analysis in this study. We also find that the impact of SUSY radiative corrections on the cesium weak charge are quite small, so that the present agreement of $Q_{W}^{\mathrm{Cs}}$ with the SM does not rule out potentially observable effects in PVES.

In contrast, the effects on $Q_{W}^{e}$ and $Q_{W}^{p}$ induced by new tree-level, $P_{R}$ violating SUSY interactions display a different behavior. Given the constraints from other precision electroweak observables, such as the Fermi constant, first row CKM unitarity, and $Q_{W}^{\mathrm{Cs}}$, one would expect $P_{R}$ violation to cause a decrease in the size of $Q_{W}^{e}$. On the other hand, the magnitude of $Q_{W}^{p}$ can change either way, with an increase being more likely. Moreover, the size of the $P_{R}$ violating corrections could be even larger than those induced by SUSY loops, particularly in the case of $Q_{W}^{p}$. Should measurements of the weak charges be consistent with this signature of $P_{R}$ violation, they could have important implications for the nature of cold dark matter (it would not be supersymmetric) and the nature of neutrinos (they would be Majorana fermions).

From either standpoint, should the PVES measurements deviate significantly from the SM predictions, one may be able to draw interesting conclusions about the character of SUSY. But what if both measurements turn out to be consistent with the SM? In this case, one would add further constraints to the possibility of $P_{R}$ violation, but only marginally constrain the MSSM parameter space based on possible loop effects. In the latter case, the impact on both $Q_{W}^{p}$ and $Q_{W}^{e}$ is dominated by $\delta \sin ^{2} \theta_{W}^{e f f}\left(q^{2}\right)^{\mathrm{SUSY}}$. Although the projected, combined statistics of the two measurements would make them more sensitive to SUSY radiative corrections than either measurement would be independently, additional precision would be advantageous. In this respect, a possible future measurement of $Q_{W}^{e}$ with a factor of two better precision than anticipated at SLAC would significantly enhance the ability of PVES to shed new light on SUSY. ${ }^{4}$

\section{ACKNOWLEDGMENTS}

We thank R. Carlini, J. Erler, R. D. McKeown, and M. Wise for useful discussions. This work is supported in part

\footnotetext{
${ }^{4}$ We thank D. Mack, P. Reimer, and P. Souder for sharing with us the possibility of such a future measurement at the Jefferson Lab.
} 
under U.S. Department of Energy contract No. DE-FG0302ER41215 (A.K. and M.J.R.-M.), No. DE-FG0300ER41132 (M.J.R.-M.), and No. DE-FG03-92-ER-40701 (S.S.). A.K. and M.J.R.-M. are supported by the National Science Foundation under grant No. PHY00-71856.

\section{APPENDIX A: COUNTERTERMS FOR THE EFFECTIVE PVES LAGRANGIAN}

The "bare" effective Lagrangian for the forward angle PVES scattering has the form:

$$
\begin{aligned}
& \mathcal{L}_{e f}=-\frac{G_{\mu}^{0}}{2 \sqrt{2}} Q_{W}^{f 0} A_{e}^{\mu 0} \times V_{\mu f}^{0}, \\
& \frac{G_{\mu}^{0}}{\sqrt{2}}=\frac{g_{0}^{2}}{8\left(M_{W}^{0}\right)^{2}}=\frac{G_{\mu}+\delta \hat{G}_{\mu}}{\sqrt{2}}, \\
& Q_{W}^{f 0}=2 I_{3}^{f}-4 Q_{f} s_{0}^{2}=2 I_{3}^{f}-4 Q_{f}\left(\hat{s}^{2}+\delta \hat{s}^{2}\right), \\
& A_{e}^{\mu 0}=\left(\bar{e} \gamma^{\mu} \gamma_{5} e\right)_{0} \equiv A_{e}^{\mu}\left(1+\frac{\delta \hat{A}_{e}}{Q_{W}^{f}}\right), \\
& V_{\mu f}^{0}=\left(\bar{f} \gamma_{\mu} f\right)_{0} \equiv V_{\mu f}\left(1+\frac{\delta \hat{V}_{f}}{Q_{W}^{f}}\right),
\end{aligned}
$$

where the bare quantities are indexed by " 0 ." Unless otherwise indicated, all higher-order contributions include both the SM and the SUSY pieces. The quantity $1 / Q_{W}^{f}$ in the parentheses in the last two lines of the above equation is explicitly factored out to make the definitions in Eq. (A4) below more convenient.

The counterterm $\delta \hat{G}_{\mu}$ is entirely determined by the muon lifetime. It can be taken from Eq. (62) in Ref. [17]:

$$
\frac{\delta \hat{G}_{\mu}}{G_{\mu}}=-\frac{\hat{\Pi}_{W W}(0)}{M_{W}^{2}}-\hat{\delta}_{V B}^{\mu},
$$

where $\hat{\Pi}_{W W}\left(q^{2}\right)$ is the $W$ boson self-energy and $\hat{\delta}_{V B}^{\mu}$ is the sum of the vertex and box corrections to the muon decay amplitude.

We use the following convention for the $Z$-fermion interaction:

$$
\begin{aligned}
V_{Z f} & =-\frac{g}{4 c} \bar{f} \gamma_{\mu}\left(g_{V}^{f}+g_{A}^{f} \gamma_{5}\right) f Z^{\mu}, \\
g_{V}^{f} & =2 I_{3}^{f}-4 Q_{f} s^{2}, \\
g_{A}^{f} & =-2 I_{3}^{f} .
\end{aligned}
$$

In this convention, the counterterms for the vector and the axial vector currents can be read off from Ref. [17]:.

\footnotetext{
${ }^{5}$ Note that Ref. [17] has the opposite sign convention for $g_{A}^{f}$.
}

$$
\begin{gathered}
\delta \hat{A}_{e}=-g_{V}^{e} \delta \hat{Z}_{A}^{e}+g_{A}^{e} \delta \hat{Z}_{V}^{e}, \\
\delta \hat{V}_{f}=g_{V}^{f} \delta \hat{Z}_{V}^{f}-g_{A}^{f} \delta \hat{Z}_{A}^{f}, \\
\delta \hat{Z}_{A}^{e, f}=\frac{1}{2}\left(\delta \hat{Z}_{L}^{e, f}-\delta \hat{Z}_{R}^{e, f}\right), \\
\delta \hat{Z}_{V}^{e, f}=\frac{1}{2}\left(\delta \hat{Z}_{L}^{e, f}+\delta \hat{Z}_{R}^{e, f}\right),
\end{gathered}
$$

where $\delta \hat{Z}_{L}^{e, f}$ and $\delta \hat{Z}_{R}^{e, f}$ are the field strength renormalization constants for left- and right-handed fermions, respectively. One can write the one-loop correction to the NC vertex as

$$
-(i g / 4 c) \delta \hat{V}_{\mu}^{f}=-(i g / 4 c) \bar{f} \gamma_{\mu}\left(\hat{G}_{V}^{f}+\gamma_{5} \hat{G}_{A}^{f}\right) f,
$$

where only the contributions that are not suppressed by powers of either the momentum transfer $\left(\sqrt{\left|q^{2}\right|} / M_{\text {SUSY }}\right)$ or the fermion mass $\left(m_{f} / M_{\text {SUSY }}\right)$ are shown. The quantities $\hat{G}_{A}^{e}$ and $\hat{G}_{V}^{f}$ represent rescaling of the vertices by the one-loop radiative corrections. They must be combined with the appropriate counterterms from Eq. (A4) to obtain the renormalized corrections:

$$
\begin{aligned}
& \hat{V}_{A}^{e}=\hat{G}_{A}^{e}+\delta \hat{A}_{e}=\hat{G}_{A}^{e}-g_{V}^{e} \delta \hat{Z}_{A}^{e}+g_{A}^{e} \delta \hat{Z}_{V}^{e}, \\
& \hat{V}_{V}^{f}=\hat{G}_{V}^{f}+\delta \hat{V}_{f}=\hat{G}_{V}^{f}+g_{V}^{f} \delta \hat{Z}_{V}^{f}-g_{A}^{f} \delta \hat{Z}_{A}^{f} .
\end{aligned}
$$

\section{APPENDIX B: DECOUPLING OF GLUINOS FROM THE WEAK CHARGE OF QUARKS}

It is sufficient to demonstrate the decoupling for one of the quark flavors (e.g. the up quark) since for other flavors the proof is identical. Consider the renormalized vector neutral current vertex for the up quark [see Eq. (A6)]:

$$
\begin{aligned}
\hat{V}_{V}^{u} & =\hat{G}_{V}^{u}+g_{V}^{u} \delta \hat{Z}_{V}^{u}-g_{A}^{u} \delta \hat{Z}_{A}^{u}, \\
\delta \hat{Z}_{V}^{u} & =\frac{\delta \hat{Z}_{L}^{u}+\delta \hat{Z}_{R}^{u}}{2}, \\
\delta \hat{Z}_{A}^{u} & =\frac{\delta \hat{Z}_{L}^{u}-\delta \hat{Z}_{R}^{u}}{2} .
\end{aligned}
$$

The gluino contributions to $\hat{G}_{V}^{u}$ are given by the graph shown in Fig. 4(b), with the neutralino replaced by the gluino. By using Eq. (C8) below with the appropriate coupling constants it is straightforward to show that

$$
\begin{aligned}
\hat{G}_{V}^{u}(\text { Gluino })= & -\frac{4}{3} \frac{\alpha_{S}}{4 \pi} \sum_{i, j}\left(\sum_{I} Z_{U}^{* I i} Z_{U}^{I j}-\frac{4}{3} \hat{s}^{2} \delta_{i j}\right)\left[g_{G L}^{* u j} g_{G L}^{u i}\right. \\
& \left.+g_{G R}^{* u j} g_{G R}^{u i}\right] V_{2}\left(M_{\tilde{g}}, m_{\widetilde{U}_{i}}, m_{\widetilde{U}_{j}}\right),
\end{aligned}
$$

where $\alpha_{S}$ is the strong coupling constant, $M_{\tilde{g}}$ is the gluino mass, $V_{2}\left(M, m_{1}, m_{2}\right)$ is defined in Eq. (C1), the couplings $Z_{U}^{I j}$ are defined in the appendixes of Ref. [11], and 


$$
\begin{aligned}
& g_{G L}^{u i}=-\sqrt{2} Z_{U}^{* 1 i}, \\
& g_{G R}^{u i}=\sqrt{2} Z_{U}^{* 4 i} .
\end{aligned}
$$

In this work, no flavor mixing in the squark sector is allowed. Therefore, $Z_{U}^{1 i}, Z_{U}^{4 i} \neq 0$ only if $i=1,4$. Since $Z_{U}$ is unitary we find

$$
g_{G L}^{* u j} g_{G L}^{u i}+g_{G R}^{* u j} g_{G R}^{u i}=2\left(Z_{U}^{1 j} Z_{U}^{* 1 i}+Z_{U}^{4 j} Z_{U}^{* 4 i}\right)=2 \delta_{i j}
$$

for $i, j=1,4$. Finally,

$$
\begin{aligned}
\hat{G}_{V}^{u}(\text { Gluino })= & -\frac{4}{3} \frac{\alpha_{S}}{2 \pi} \sum_{i=1,4}\left(\left|Z_{U}^{1 i}\right|^{2}\right. \\
& \left.-\frac{4}{3} s^{2}\right) V_{2}\left(M_{\tilde{g}}, m_{\tilde{U}_{i}}, m_{\tilde{U}_{i}}\right) \\
= & -\frac{4}{3} \frac{\alpha_{S}}{2 \pi}\left\{\frac { g _ { V } ^ { u } } { 2 } \left[V_{2}\left(M_{\tilde{g}}, m_{\tilde{U}_{1}}, m_{\tilde{U}_{1}}\right)\right.\right. \\
& \left.+V_{2}\left(M_{\tilde{g}}, m_{\tilde{U}_{4}}, m_{\tilde{U}_{4}}\right)\right]-\frac{g_{A}^{u}}{2}(1 \\
& \left.-2\left|Z_{U}^{14}\right|^{2}\right)\left[V_{2}\left(M_{\tilde{g}}, m_{\tilde{U}_{1}}, m_{\widetilde{U}_{1}}\right)\right. \\
& \left.\left.-V_{2}\left(M_{\tilde{g}}, m_{\tilde{U}_{4}}, m_{\tilde{U}_{4}}\right)\right]\right\},
\end{aligned}
$$

where the closure property Eq. (B4) was used together with

$$
\begin{aligned}
& g_{V}^{u}=2\left(I_{3}^{u}-2 Q^{u} \hat{s}^{2}\right)=1-\frac{8}{3} \hat{s}^{2}, \\
& g_{A}^{u}=-2 I_{3}^{u}=-1 .
\end{aligned}
$$

On the other hand, the gluino-induced wave function renormalization constants of the up quark have the form

$$
\begin{aligned}
& \delta \hat{Z}_{V}^{u}(\text { Gluino })=\frac{4}{3} \frac{\alpha_{S}}{8 \pi} \sum_{i}\left(\left|g_{G L}^{u i}\right|^{2}+\left|g_{G R}^{u i}\right|^{2}\right) F_{1}\left(m_{\widetilde{U}_{i}}, M_{\tilde{g}}, 0\right), \\
& \delta \hat{Z}_{A}^{u}(\text { Gluino })=\frac{4}{3} \frac{\alpha_{S}}{8 \pi} \sum_{i}\left(\left|g_{G L}^{u i}\right|^{2}-\left|g_{G R}^{u i}\right|^{2}\right) F_{1}\left(m_{\tilde{U}_{i}}, M_{\tilde{g}}, 0\right),
\end{aligned}
$$

where $F_{1}\left(m_{1}, m_{2}, m_{3}\right)$ is given by

$$
\begin{aligned}
F_{1}\left(m_{1}, m_{2}, m_{3}\right)= & \int_{0}^{1} x \ln \left\{\left[x m_{1}^{2}+(1-x) m_{2}^{2}-x(1\right.\right. \\
& \left.\left.-x) m_{3}^{2}\right] / \mu^{2}\right\} .
\end{aligned}
$$

Note that according to Eq. (C1), $F_{1}\left(m_{1}, m_{2}, 0\right)$ $\equiv V_{2}\left(m_{2}, m_{1}, m_{1}\right)$. Using Eqs. (B3) and (B4) we find

$$
\begin{aligned}
\delta \hat{Z}_{V}^{u}(\text { Gluino })= & \frac{4}{3} \frac{\alpha_{S}}{4 \pi}\left[V_{2}\left(M_{\tilde{g}}, m_{\widetilde{U}_{1}}, m_{\widetilde{U}_{1}}\right)\right. \\
& \left.+V_{2}\left(M_{\tilde{g}}, m_{\tilde{U}_{4}}, m_{\widetilde{U}_{4}}\right)\right], \\
\delta \hat{Z}_{A}^{u}(\text { Gluino })= & \frac{4}{3} \frac{\alpha_{S}}{4 \pi}\left(1-2\left|Z_{U}^{14}\right|^{2}\right) \\
& \times\left[V_{2}\left(M_{\tilde{g}}, m_{\tilde{U}_{1}}, m_{\tilde{U}_{1}}\right)\right. \\
& \left.-V_{2}\left(M_{\tilde{g}}, m_{\tilde{U}_{4}}, m_{\widetilde{U}_{4}}\right)\right] .
\end{aligned}
$$

After substitution of Eqs. (B5) and (B9) into Eq. (B1) the gluino corrections to the vector neutral current vertex of the up quark cancel exactly. Therefore, gluino loops do not renormalize the weak charge of the up quark.

\section{APPENDIX C: COMPLETE EXPRESSIONS FOR FEYNMAN DIAGRAMS}

In this appendix we list analytical expressions for all SUSY one-loop vertex and box Feynman diagrams that contribute to PV electron scattering. The complete expressions for remaining diagrams (see Sec. III) as well as the Feynman rules are given in the appendixes of Ref. [11]. We use the capitalized letters $I$ and $J$ to denote the family index for quarks and leptons $(I, J=1, \ldots, 3)$, small letters $i$ and $j$ to denote the index for squarks and sleptons $(i, j=1, \ldots, 6$ except for sneutrino, when $i, j=1, \ldots, 3)$, and small letters $p$ and $n$ to denote the index for the neutralinos $(p, n$ $=1, \ldots, 4)$ and charginos $(p, n=1,2)$.

\section{Vertex corrections}

The Feynman diagrams are shown in Figs. 3 and 4. Let us start with the corrections to the $e-e-Z$ vertex. The loop integral functions $V_{1}\left(m_{1}, m_{2}, m_{3}\right)$ and $V_{2}\left(m_{1}, m_{2}, m_{3}\right)$ are defined as

$$
\begin{aligned}
& V_{1}\left(M, m_{1}, m_{2}\right)=\int_{0}^{1} d x \int_{0}^{1} d y \frac{y}{D_{3}\left(M, m_{1}, m_{2}\right)}, \\
& V_{2}\left(M, m_{1}, m_{2}\right)=\int_{0}^{1} d x \int_{0}^{1} d y y \ln \left[D_{3}\left(M, m_{1}, m_{2}\right) / \mu^{2}\right], \\
& D_{3}\left(M, m_{1}, m_{2}\right)=(1-y) M^{2}+y\left[(1-x) m_{1}^{2}+x m_{2}^{2}\right],
\end{aligned}
$$

where $\mu$ is the renormalization scale. Explicitly

$$
\begin{gathered}
\frac{m_{1}^{2} \ln \frac{m_{1}^{2}}{M^{2}}}{\left(M^{2}-m_{1}^{2}\right)\left(m_{2}^{2}-m_{1}^{2}\right)} \\
+\frac{m_{2}^{2} \ln \frac{m_{2}^{2}}{M^{2}}}{\left(M^{2}-m_{2}^{2}\right)\left(m_{1}^{2}-m_{2}^{2}\right)},
\end{gathered}
$$




$$
\begin{aligned}
V_{2}\left(M, m_{1}, m_{2}\right)= & \frac{1}{4}\left[2 \ln M^{2}-3\right. \\
& +\frac{2 m_{1}^{4}}{\left(M^{2}-m_{1}^{2}\right)\left(m_{2}^{2}-m_{1}^{2}\right)} \ln \frac{m_{1}^{2}}{M^{2}} \\
& +\frac{2 m_{2}^{4}}{\left(M^{2}-m_{2}^{2}\right)\left(m_{1}^{2}-m_{2}^{2}\right)} \ln \frac{m_{2}^{2}}{M^{2}} \\
& \left.-2 \ln \mu^{2}\right] .
\end{aligned}
$$

We have $\left[\hat{P}_{L}=\left(1-\gamma_{5}\right) / 2, \hat{P}_{R}=\left(1+\gamma_{5}\right) / 2\right]$

$$
\begin{aligned}
& \delta \hat{V}_{\mu}^{e(a)}=-\frac{\alpha}{2 \pi} \sum_{i, j, p}\left(\sum_{I} Z_{\nu}^{* I i} Z_{\nu}^{I j}\right. \\
& \left.-2 Q_{\nu} \hat{s}^{2} \delta_{i j}\right) V_{2}\left(m_{\chi_{p}^{+}}, m_{\tilde{\nu}_{i}}, m_{\tilde{\nu}_{j}}\right) \bar{e} \gamma_{\mu}\left[g_{L}^{* e j p} g_{L}^{e i p} \hat{P}_{L}\right. \\
& \left.+g_{R}^{* e j p} g_{R}^{e i p} \hat{P}_{R}\right] e .
\end{aligned}
$$

Note that $Q_{\nu}=0$ and $Z_{\nu}^{i j}$ is a unitary $3 \times 3$ matrix. Therefore, one identically has $\Sigma_{I} Z_{\nu}^{* I i} Z_{\nu}^{I j}-2 Q_{\nu} \hat{s}^{2} \delta_{i j}=\delta_{i j}$. The explicit form is kept so that the down quark neutral current vertex may be easily obtained by the replacement $e \rightarrow d$ (with $Z_{L}$ $\rightarrow Z_{D}$ ) and $\nu \rightarrow u$.

$$
\begin{aligned}
& \delta \hat{V}_{\mu}^{e(b)}=\frac{\alpha}{2 \pi} \sum_{i, j, p}\left(\sum_{I} Z_{L}^{* I i} Z_{L}^{I j}\right. \\
& \left.+2 Q_{e} \hat{s}^{2} \delta_{i j}\right) V_{2}\left(m_{\chi_{p}^{0}}, m_{\tilde{L}_{i}}, m_{\tilde{L}_{j}}\right) \bar{e} \gamma_{\mu}\left[g_{0 L}^{* e j p} g_{0 L}^{e i p} \hat{P}_{L}\right. \\
& \left.+g_{0 R}^{* e j p} g_{0 R}^{e i p} \hat{P}_{R}\right] e, \\
& \delta \hat{V}_{\mu}^{e(c)}=\frac{\alpha}{2 \pi} \sum_{i, p, n} \bar{e} \gamma_{\mu}\left\{\left[O_{p n}^{R^{\prime}} g_{L}^{* e i n} g_{L}^{e i p} \hat{P}_{L}\right.\right. \\
& \left.+O_{p n}^{L^{\prime}} g_{R}^{* e i n} g_{R}^{e i p} \hat{P}_{R}\right] 2 m_{\chi_{p}^{+}} m_{\chi_{n}^{+}} V_{1}\left(m_{\tilde{\nu}_{i}}, m_{\chi_{p}^{+}}, m_{\chi_{n}^{+}}\right) \\
& -\left[O_{p n}^{L^{\prime}} g_{L}^{* e i n} g_{L}^{e i p} \hat{P}_{L}+O_{p n}^{R^{\prime}} g_{R}^{* e i n} g_{R}^{\text {eip }} \hat{P}_{R}\right] \\
& \left.\times\left[1+2 V_{2}\left(m_{\tilde{\nu}_{i}}, m_{\chi_{p}^{+}}, m_{\chi_{n}^{+}}\right)\right]\right\} e, \\
& \delta \hat{V}_{\mu}^{e(d)}=-\frac{\alpha}{2 \pi} \sum_{p, n, i} \bar{e} \gamma_{\mu}\left\{\left[O_{n p}^{L^{\prime \prime}} g_{0 L}^{* e i n} g_{0 L}^{e i p} \hat{P}_{L}\right.\right.
\end{aligned}
$$

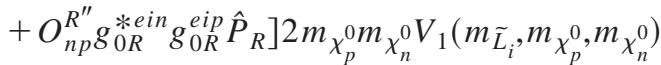

$$
\begin{aligned}
& -\left[O_{n p}^{R^{\prime \prime}} g_{0 L}^{* e i n} g_{0 L}^{e i p} \hat{P}_{L}+O_{n p}^{L^{\prime \prime}} g_{0 R}^{* e i n} g_{0 R}^{e i p} \hat{P}_{R}\right] \\
& \left.\times\left[1+2 V_{2}\left(m_{\tilde{L}_{i}}, m_{\chi_{p}^{0}}, m_{\chi_{n}^{0}}\right)\right]\right\} e .
\end{aligned}
$$

The vector and axial vector pieces can be readily read off from the above formulas. The radiative corrections to the up quark neutral current vertex are as follows (see Fig. 4):

$$
\begin{aligned}
\delta \hat{V}_{\mu}^{u(a)}= & \frac{\alpha}{2 \pi} \sum_{i, j, p}\left(\sum_{I} Z_{D}^{* I i} Z_{D}^{I j}\right. \\
& \left.-\frac{2}{3} \hat{s}^{2} \delta_{i j}\right) V_{2}\left(m_{\chi_{p}^{+}}, m_{\tilde{D}_{i}}, m_{\tilde{D}_{j}}\right) \bar{u} \gamma_{\mu}\left[g_{L}^{* u j p} g_{L}^{u i p} \hat{P}_{L}\right. \\
& \left.+g_{R}^{* u j p} g_{R}^{u i p} \hat{P}_{R}\right] u, \\
\delta \hat{V}_{\mu}^{u(b)}= & -\frac{\alpha}{2 \pi} \sum_{i, j, p}\left(\sum_{I} Z_{U}^{* I i} Z_{U}^{I j}\right. \\
& \left.-\frac{4}{3} \hat{s}^{2} \delta_{i j}\right) V_{2}\left(m_{\chi_{p}^{0}}, m_{\tilde{U}_{i}}, m_{\tilde{U}_{j}}\right) \bar{u} \gamma_{\mu}\left[g_{0 L}^{* u j p} g_{0 L}^{u i p} \hat{P}_{L}\right. \\
& \left.+g_{0 R}^{* u j p} g_{0 R}^{u i p} \hat{P}_{R}\right] u, \\
\delta \hat{V}_{\mu}^{u(c)}= & -\frac{\alpha}{2 \pi} \sum_{i, p, n} \bar{u}_{\mu}\left\{\left[O_{n p}^{L^{\prime}} g_{L}^{* u i n} g_{L}^{u i p} \hat{P}_{L}\right.\right. \\
& \left.+O_{n p}^{R^{\prime}} g_{R}^{* u i n} g_{R}^{u i p} \hat{P}_{R}\right] 2 m_{\chi_{p}^{+}} m_{\chi_{n}^{+}} V_{1}\left(m_{\tilde{D}_{i}}, m_{\chi_{p}^{+}}, m_{\chi_{n}^{+}}\right) \\
& -\left[O_{n p}^{R^{\prime}} g_{L}^{* u i n} g_{L}^{u i p} \hat{P}_{L}+O_{n p}^{L^{\prime}} g_{R}^{* u i n} g_{R}^{u i p} \hat{P}_{R}\right] \\
& \left.\times\left[1+2 V_{2}\left(m_{\tilde{D}_{i}}, m_{\chi_{p}^{+}}, m_{\chi_{n}^{+}}\right)\right]\right\} u,
\end{aligned}
$$

$$
\begin{aligned}
\delta \hat{V}_{\mu}^{u(d)}= & -\frac{\alpha}{2 \pi} \sum_{p, n, i} \bar{u} \gamma_{\mu}\left\{\left[O_{n p}^{L^{\prime \prime}} g_{0 L}^{* u i n} g_{0 L}^{u i p} \hat{P}_{L}\right.\right. \\
& \left.+O_{n p}^{R^{\prime \prime}} g_{0 R}^{* u i n} g_{0 R}^{u i p} \hat{P}_{R}\right] 2 m_{\chi_{p}^{0}} m_{\chi_{n}^{0}} V_{1}\left(m_{\widetilde{U}_{i}}, m_{\chi_{p}^{0}}, m_{\chi_{n}^{0}}\right) \\
& -\left[O_{n p}^{R^{\prime \prime}} g_{0 L}^{* u i n} g_{0 L}^{u i p} \hat{P}_{L}+O_{n p}^{L^{\prime \prime}} g_{0 R}^{* u i n} g_{0 R}^{u i p} \hat{P}_{R}\right] \\
& \left.\times\left[1+2 V_{2}\left(m_{\widetilde{U}_{i}}, m_{\chi_{p}^{0}}, m_{\chi_{n}^{0}}\right)\right]\right\} u .
\end{aligned}
$$

\section{Anapole moment corrections}

Using formulas in the Appendix of Ref. [11] we find for $F_{A}^{e}(0)$ of the electron:

$$
\begin{aligned}
F_{A}^{e}(0)= & F_{A}^{(a)}(0)+F_{A}^{(b)}(0), \\
F_{A}^{(a)}(0)= & -\frac{\alpha M_{Z}^{2}}{48 \pi} \sum_{i, p}\left|g_{L}^{e i p}\right|^{2} \\
& \times \int_{0}^{1} \frac{x^{2}(x-3) d x}{(1-x) m_{\tilde{\nu}_{i}}^{2}+x m_{\chi_{p}^{+}}^{2}}, \\
F_{A}^{(b)}(0)= & \frac{\alpha M_{Z}^{2}}{48 \pi} \sum_{i, p}\left(\left|g_{0 L}^{e i p}\right|^{2}-\left|g_{0 R}^{e i p}\right|^{2}\right) \\
& \times \int_{0}^{1} \frac{x^{3} d x}{(1-x) m_{\chi_{p}^{0}}^{2}+x m_{\tilde{L}_{i}}^{2}} .
\end{aligned}
$$




\section{Box graphs}

Let us introduce the following notation:

$$
\begin{aligned}
& L_{f i}=\bar{f}\left(p_{i}^{\prime}\right) \gamma_{\mu}\left(1-\gamma_{5}\right) f\left(p_{i}\right), \\
& R_{f i}=\bar{f}\left(p_{i}^{\prime}\right) \gamma_{\mu}\left(1+\gamma_{5}\right) f\left(p_{i}\right) .
\end{aligned}
$$

As explicitly shown below, each box graph has the following structure:

$$
\begin{aligned}
M_{B o x}^{e f}= & i \frac{G_{\mu}}{\sqrt{2}}\left(A^{e f} L_{f 2} \times L_{e 1}+B^{e f} R_{f 2} \times R_{e 1}+C^{e f} R_{f 2} \times L_{e 1}\right. \\
& \left.+D^{e f} L_{f 2} \times R_{e 1}\right) .
\end{aligned}
$$

To study the effects of the parity-violating electron scattering we need to pick only the term that has axial vector current on the projectile side $e 1$ and the vector current on the target side $f 2$. Therefore, it is easily seen that the box diagram contribution to $C_{1 f}$ is [see Eq. (11)]

$$
\hat{\delta}_{B o x}^{e f}=-2\left(-A^{e f}+B^{e f}-C^{e f}+D^{e f}\right)
$$

in the above notation. The explicit expressions for the ee box graphs in Fig. 5 are given below:

$$
\begin{aligned}
& \delta M_{B o x}^{e e(a)}=i \frac{G_{\mu}}{\sqrt{2}} \frac{\alpha M_{W}^{2} \hat{s}^{2}}{4 \pi} \sum_{n, p, i, j}\left\{\left[g_{0 L}^{* e j n} g_{0 L}^{e j p} g_{0 L}^{* e i n} g_{0 L}^{e i p} L_{e 2} \times L_{e}\right.\right. \\
& +g_{0 R}^{* e j n} g_{0 R}^{e j p} g_{0 R}^{* e i n} g_{0 R}^{e i p} R_{e 2}
\end{aligned}
$$

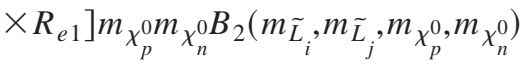

$$
\begin{aligned}
& +\left[g_{0 R}^{* e j n} g_{0 R}^{e j p} g_{0 L}^{* e i n} g_{0 L}^{e i p} R_{e 2} \times L_{e 1}\right. \\
& +g_{0 L}^{* e j n} g_{0 L}^{e j p} g_{0 R}^{* e i n} g_{0 R}^{e i p} L_{e 2} \\
& \left.\left.\times R_{e 1}\right] B_{1}\left(m_{\tilde{L}_{i}}, m_{\tilde{L}_{j}}, m_{\chi_{p}^{0}}, m_{\chi_{n}^{0}}\right)\right\}, \\
& \delta M_{B o x}^{e e(b)}=-i \frac{G_{\mu}}{\sqrt{2}} \frac{\alpha M_{W}^{2} \hat{s}^{2}}{4 \pi} \sum_{n, p, i, j}\left\{\left[g_{0 L}^{* e j n} g_{0 L}^{e j p} g_{0 L}^{e i n} g_{0 L}^{* e i p} L_{e 2}\right.\right. \\
& \times L_{e 1}+g_{0 R}^{* e j n} g_{0 R}^{e j p} g_{0 R}^{e i n} g_{0 R}^{* e i p} R_{e 2} \\
& \left.\times R_{e 1}\right] B_{1}\left(m_{\tilde{L}_{i}}, m_{\tilde{L}_{j}}, m_{\chi_{p}^{0}}, m_{\chi_{n}^{0}}\right) \\
& +\left[g_{0 R}^{* e j n} g_{0 R}^{e j p} g_{0 L}^{e i n} g_{0 L}^{* e i p} R_{e 2} \times L_{e 1}\right. \\
& +g_{0 L}^{* e j n} g_{0 L}^{e j p} g_{0 R}^{e i n} g_{0 R}^{* e i p} L_{e 2} \\
& \left.\left.\times R_{e 1}\right] m_{\chi_{p}^{0}} m_{\chi_{n}^{0} B_{2}}\left(m_{\tilde{L}_{i}}, m_{\tilde{L}_{j}}, m_{\chi_{p}^{0}}, m_{\chi_{n}^{0}}\right)\right\}, \\
& \delta M_{B o x}^{e e(c)}=-i \frac{G_{\mu}}{\sqrt{2}} \frac{\alpha M_{W}^{2} \hat{s}^{2}}{4 \pi} \sum_{n, p, i, j} g_{L}^{* e i p} g_{L}^{e i n} g_{L}^{e j p} g_{L}^{* e j n} L_{e 2} \\
& \times L_{e 1} B_{1}\left(m_{\tilde{\nu}_{i}}, m_{\tilde{\nu}_{j}}, m_{\chi_{p}^{+}}, m_{\chi_{n}^{+}}\right) .
\end{aligned}
$$

In all the above formulas the following functions are used:

$$
\begin{gathered}
B_{1}\left(M_{1}, M_{2}, m_{1}, m_{2}\right) \\
=\int_{0}^{1} d x \int_{0}^{1} d y \int_{0}^{1} d z \frac{z(1-z)}{D_{4}\left(M_{1}, M_{2}, m_{1}, m_{2}\right)}, \\
B_{2}\left(M_{1}, M_{2}, m_{1}, m_{2}\right) \\
=\int_{0}^{1} d x \int_{0}^{1} d y \int_{0}^{1} d z \frac{z(1-z)}{D_{4}^{2}\left(M_{1}, M_{2}, m_{1}, m_{2}\right)}, \\
D_{4}\left(M_{1}, M_{2}, m_{1}, m_{2}\right) \\
=z\left[(1-x) M_{1}^{2}+x M_{2}^{2}\right]+(1-z)\left[\left(y m_{1}^{2}+(1-y) m_{2}^{2}\right] .\right.
\end{gathered}
$$

Explicitly,

$$
\begin{aligned}
B_{1}\left(M_{1}, M_{2}, m_{1}, m_{2}\right)= & \frac{m_{1}^{4} \ln \frac{m_{1}^{2}}{M_{2}^{2}}}{2\left(m_{1}^{2}-M_{1}^{2}\right)\left(m_{1}^{2}-m_{2}^{2}\right)\left(m_{1}^{2}-M_{2}^{2}\right)} \\
& +\frac{m_{2}^{4} \ln \frac{m_{2}^{2}}{M_{2}^{2}}}{2\left(m_{2}^{2}-m_{1}^{2}\right)\left(m_{2}^{2}-M_{1}^{2}\right)\left(m_{2}^{2}-M_{2}^{2}\right)} \\
& +\frac{M_{1}^{4} \ln \frac{M_{1}^{2}}{M_{2}^{2}}}{B_{2}\left(M_{1}^{2}, M_{2}^{2}, m_{1}, m_{2}\right)=} \\
& +\frac{\left.m_{1}^{2}-m_{2}^{2}\right)\left(M_{1}^{2}-M_{2}^{2}\right)}{\left(m_{1}^{2}-M_{1}^{2}\right)\left(m_{1}^{2}-m_{2}^{2}\right)\left(m_{1}^{2}-M_{2}^{2}\right)} \\
& +\frac{m_{1}^{2} \ln \frac{M_{2}^{2}}{m_{2}^{2}}}{\left(m_{2}^{2}-m_{1}^{2}\right)\left(m_{2}^{2}-M_{1}^{2}\right)\left(m_{2}^{2}-M_{2}^{2}\right)} \\
M_{1}^{2} \ln \frac{M_{2}^{2}}{M_{1}^{2}} & \left.m_{2}^{2}\right)\left(M_{1}^{2}-M_{2}^{2}\right)
\end{aligned}
$$

The box graphs for the electron-down quark scattering are easily obtained from the above expressions by replacing the target electron $e 2$ with the down quark. Also, all quantities that have the running index $j$ re to be replaced with the corresponding quantities for the first generation down squarks: $g_{0 L}^{e j n} \rightarrow g_{0 L}^{d j n}$, etc.

The box graphs for the electron-up quark scattering are shown in Fig. 6. The graphs 6(a) and 6(b) are easily obtained from the corresponding graphs for the ee scattering by replacing all quantities that have the running index $i$ with the corresponding quantities for the first up generation squarks: $g_{0 L}^{\text {ein }} \rightarrow g_{0 L}^{\text {uin }}$, etc. The result for the last graph 6(c) is 


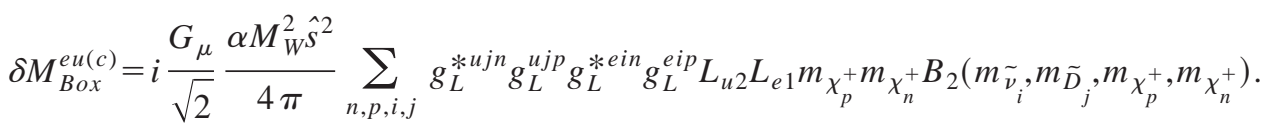

[1] C.Y. Prescott et al., Phys. Lett. 77B, 347 (1978); 84B, 524 (1979).

[2] S.C. Bennett and C.E. Wieman, Phys. Rev. Lett. 82, 2484 (1999); C.S. Wood et al., Science 275, 1759 (1997).

[3] NuTev Collaboration, G.P. Zeller et al., Phys. Rev. Lett. 88, 091802 (2002).

[4] E.W. Hughes, K. Kumar, and P.A. Souder, SLAC Experiment E-158.

[5] R. Carlini, J.M. Finn, S. Kowalski, and S. Page, JLab Experiment E-02-020.

[6] J. Erler, M.J. Ramsey-Musolf, and A. Kurylov, Phys. Rev. D 68, 016006 (2003).

[7] G. Jungman, M. Kamionkowski, and K. Griest, Phys. Rep. 267, 195 (1996).

[8] H.E. Haber and G.L Kane, Phys. Rep. 117, 74 (1985).

[9] A. Kurylov and M.J. Ramsey-Musolf, Phys. Rev. Lett. 88, 071804 (2002).

[10] A. Czarnecki and W. Marciano, Phys. Rev. D 53, 1066 (1996).

[11] A. Kurylov, M.J. Ramsey-Musolf, and S. Su, Nucl. Phys. B (to be published), hep-ph/0301208.

[12] Particle Data Group, K. Hagiwara et al., Phys. Rev. D 66, 010001 (2002).

[13] J. Rosiek, Phys. Rev. D 41, 3464 (1990); hep-ph/9511250.

[14] See, e.g., Perspectives on Supersymmetry, edited by G.L. Kane
(World Scientific, Singapore, 1998).

[15] J. Erler and D.M. Pierce, Nucl. Phys. B526, 53 (1998).

[16] W. Siegel, Phys. Lett. 84B, 193 (1979).

[17] Damien M. Pierce, hep-ph/9805497.

[18] W.J. Marciano and A. Sirlin, Phys. Rev. D 22, 2695 (1980).

[19] G. Degrassi, B.A. Kniehl, and A. Sirlin, Phys. Rev. D 48, 3963 (1993).

[20] H.E. Haber, hep-ph/9306207; S. Heinemeyer and G. Weiglein, hep-ph/0102317.

[21] M.J. Musolf and B.R. Holstein, Phys. Rev. D 43, 2956 (1991).

[22] M.J. Ramsey-Musolf, Phys. Rev. D 62, 056009 (2000).

[23] Muon g-2 Collaboration, G.W. Bennett et al., Phys. Rev. Lett. 89, 101804 (2002).

[24] M.J. Ramsey-Musolf, Phys. Rev. C 60, 015501 (1999).

[25] A.I. Milstein, O.P. Sushkov, and I.S. Terekhov, Phys. Rev. A 67, 062103 (2003).

[26] I.S. Towner and J.C. Hardy, in Proceedings of the Fifth International WEIN Symposium: Physics Beyond the Standard Model, edited by P. Herczeg et al. (World Scientific, Singapore, 1999), p. 338.

[27] G. Czapek et al., Phys. Rev. Lett. 70, 17 (1993); D.I. Britton et al., ibid. 68, 3000 (1992).

[28] W.J. Marciano, Phys. Rev. D 60, 093006 (1999). 